\title{
Discrete Fractional Fourier Transform Based on New Nearly Tridiagonal Commuting Matrices
}

\author{
Soo-Chang Pei, Fellow, IEEE, Wen-Liang Hsue, and Jian-Jiun Ding
}

\begin{abstract}
Based on discrete Hermite-Gaussian-like functions, a discrete fractional Fourier transform (DFRFT), which provides sample approximations of the continuous fractional Fourier transform, was defined and investigated recently. In this paper, we propose a new nearly tridiagonal matrix, which commutes with the discrete Fourier transform (DFT) matrix. The eigenvectors of the new nearly tridiagonal matrix are shown to be DFT eigenvectors, which are more similar to the continuous Hermite-Gaussian functions than those developed before. Rigorous discussions on the relations between the eigendecomposition of the newly proposed nearly tridiagonal matrix and the DFT matrix are described. Furthermore, by appropriately combining two linearly independent matrices that both commute with the DFT matrix, we develop a method to obtain DFT eigenvectors even more similar to the continuous Hermite-Gaussian functions (HGFs). Then, new versions of DFRFT produce their transform outputs closer to the samples of the continuous fractional Fourier transform, and their applications are described. Related computer experiments are performed to illustrate the validity of the works in this paper.
\end{abstract}

Index Terms-Discrete fractional Fourier transform (DFRFT), discrete Fourier transform, Hermite-Gaussian functions.

\section{INTRODUCTION}

$\mathbf{T}$ RANSFORM operators have been frequently exploited for signal analysis, compression, and other applications in signal processing area. One of the most important transform operators is the Fourier transform. The continuous fractional Fourier transform (FRT) is a generalization of the continuous Fourier transform [8]-[10]. Some of the possible applications of the FRT are optical signal processing, quantum mechanics, time-frequency representation, optimal filtering [8]-[12], etc. Because of the importance of the FRT, the discrete fractional Fourier transform (DFRFT), which can be used for digitally computing the FRT, was defined and investigated recently [2]-[7], [21]. The basic requirements of a definition of the DFRFT are [2]-[4] 1) additive and 2) approximating the continuous FRT.

Most of the recently proposed DFRFTs in the open literature [2]-[4] were based on a nearly tridiagonal matrix, which commutes with the DFT matrix and was first introduced by Dickinson and Steiglitz [13]. For example, in [2] and [3], Pei et al.

Manuscript received July 21, 2004; revised October 11, 2005. The associate editor coordinating the review of this manuscript and approving it for publication was Dr. Steven J. Grant. This work was supported by the National Science Council of Taiwan, R.O.C., under contracts 93-2219-E-002-004 and NSC 93-2752-E-002-006-PAE

The authors are with the Department of Electrical Engineering and the Graduate Institute of Communication Engineering, National Taiwan University, Taipei, Taiwan, 10617, R.O.C. (e-mail: pei@cc.ee.ntu.edu.tw; d92942015@ntu.edu.tw; djj1@ms63.hinet.net).

Digital Object Identifier 10.1109/TSP.2006.879313 found that the eigenvectors of the nearly tridiagonal commuting matrix of the DFT matrix proposed by Dickinson et al. [13] are discrete Hermite-Gaussian-like functions. Based on these discrete Hermite-Gaussian-like functions, Pei et al. proposed an eigendecompostion-based definition of the DFRFT, of which its transform results approximate samples of the continuous fractional Fourier transform. In [4], Candan et al. consolidated and provided rigorous discussions on the DFRFT proposed by Pei et al.

In [1], Grünbaum introduced an exactly tridiagonal matrix that commutes with the centered discrete Fourier transform matrix of even size. Grünbaum also showed that his exactly tridiagonal matrix can be viewed as the discrete analogue of the second-order Hermite differential operator [1]. Inspired by the work of Grünbaum, we propose in this paper a new nearly tridiagonal matrix commuting with the ordinary DFT matrix of any size, even or odd. Most of the eigenvectors of this new nearly tridiagonal commuting matrix will be better sample approximations of the continuous Hermite-Gaussian functions (HGFs), in the sense of smaller norms of approximation error vectors, than those of the Dickinson and Steiglitz matrix. Based on the eigendecomposition of the new nearly tridiagonal matrix, we will provide a new definition of DFRFT, which is closer to the continuous FRT. Furthermore, it will be shown that the new nearly tridiagonal matrix can be linearly combined with the Dickinson and Steiglitz matrix to generate several matrices which also commute with the DFT matrix and have Hermite-Gaussian-like eigenvectors even closer to the continuous HGFs.

\section{PRELIMINARIES}

\section{A. Continuous Fractional Fourier Transform}

The $a^{\text {th }}$-order continuous FRT of $x(t)$ is defined as [8], [9]

$$
X_{a}(u)=\int_{-\infty}^{+\infty} x(t) K_{a}(t, u) d t
$$

where the transform kernel $K_{a}(t, u)$ is given by

$$
K_{a}(t, u)=\left\{\begin{array}{l}
\sqrt{1-j \cot \alpha} e^{j \pi\left(t^{2} \cot \alpha-2 t u \csc (\alpha)+u^{2} \cot \alpha\right)}, \\
\quad \text { if } \alpha \text { is not a multiple of } \pi \\
\delta(t-u), \\
\quad \text { if } \alpha \text { is a multiple of } 2 \pi \\
\delta(t+u), \\
\text { if } \alpha+\pi \text { is a multiple of } 2 \pi
\end{array}\right.
$$


in which $\alpha=a \pi / 2$. It is known that the transform kernel $K_{a}(t, u)$ can also be written as [9], [10]

$$
K_{a}(t, u)=\sum_{n=0}^{\infty} \exp (-j n a \pi / 2) \cdot \Psi_{n}(t) \Psi_{n}(u)
$$

where

$$
\Psi_{n}(t)=\frac{2^{\frac{1}{4}}}{\sqrt{2^{n} n !}} H_{n}(\sqrt{2 \pi} \cdot t) e^{-\pi \cdot t^{2}}
$$

is the $n^{\text {th }}$-order HGF with $H_{n}$ being the $n^{\text {th }}$-order Hermite polynomial.

\section{B. DFRFT Based on Commuting Matrix $\mathbf{S}$}

In this subsection, the definition of the DFRFT discussed in [2]-[4] is briefly summarized. The $N$-point DFT matrix $\mathbf{F}$ is defined by

$$
\mathbf{F}_{k n}=\frac{1}{\sqrt{N}} e^{-j \frac{2 \pi}{N} k n}, \quad 0 \leq k, n \leq N-1 .
$$

It is known that the DFT matrix has only four distinct eigenvalues $1,-1, j$, and $-j$ [14]. In [13], Dickinson and Steiglitz introduced a nearly tridiagonal matrix $\mathbf{S}$ as shown in (6), which commutes with the DFT matrix F. See (6) shown at the bottom of the page, where $\omega=2 \pi / N$. That is, with $\mathbf{S}$ defined above, $\mathbf{S F}=\mathbf{F S}$. Therefore, the DFT matrix $\mathbf{F}$ and the above matrix $\mathbf{S}$ share a common eigenvector set, and we can find the eigenvectors of $\mathbf{F}$ from those of the matrix $\mathbf{S}$ [4]. Furthermore, the matrix $\mathbf{S}$ has the following properties [2]:

1) the eigenvectors of $\mathbf{S}$ approximate samples of the continuous HGFs, and thus eigenvectors of $\mathbf{S}$ can be seen as the discrete Hermite-Gaussian-like functions;

2) the eigenvector of $\mathbf{S}$ with $k$ zero-crossings can approximate the $k^{\text {th }}$-order continuous HGF.
In [2] and [3], Pei et al. defined the $a^{\text {th }}$-order DFRFT matrix $\mathbf{F}_{\mathrm{S}}^{a}$ by

$$
\mathbf{F}_{\mathbf{S}}^{a}=\mathbf{V D}^{a} \mathbf{V}^{T}= \begin{cases}\sum_{k=0}^{N-1} e^{-j \frac{\pi}{2} k a} \mathbf{v}_{k} \mathbf{v}_{k}^{T}, & \text { for } N \text { odd } \\ \sum_{k=0}^{N-2} e^{-j \frac{\pi}{2} k a} \mathbf{v}_{k} \mathbf{v}_{k}^{T} & \\ +e^{-j \frac{\pi}{2} N a} \mathbf{v}_{N} \mathbf{v}_{N}^{T}, & \text { for } N \text { even }\end{cases}
$$

where $\mathbf{V}=\left[\mathbf{v}_{0}\left|\mathbf{v}_{\mathbf{1}}\right| \ldots\left|\mathbf{v}_{N-\mathbf{2}}\right| \mathbf{v}_{N-1}\right]$ for odd $N$ and $\mathbf{V}=$ $\left[\mathbf{v}_{\mathbf{0}}\left|\mathbf{v}_{\mathbf{1}}\right| \ldots\left|\mathbf{v}_{\boldsymbol{N}-\mathbf{2}}\right| \mathbf{v}_{\boldsymbol{N}}\right]$ for even $N, \mathbf{D}$ is a diagonal matrix with its diagonal entries corresponding to the eigenvalues for each column eigenvectors in $\mathbf{V}$, and $\mathbf{v}_{k}$ is the $k^{\text {th }}$-order discrete Hermite-Gaussian-like function with $k$ zero-crossings and is obtained from the corresponding normalized eigenvector of $\mathbf{S}$. The S-based DFRFT of input data $\mathbf{y}$ is obtained by $\mathbf{y}_{\boldsymbol{a}}=\mathbf{F}_{\mathrm{S}}^{\boldsymbol{a}} \mathbf{y}$.

\section{New Nearly Tridiagonal Commuting Matrix T}

In this section, we propose a novel nearly tridiagonal matrix which commutes with the ordinary DFT matrix $\mathbf{F}$ of any size. Moreover, we will demonstrate that using the eigenvectors of the new matrix to approximate the samples of continuous HGFs is always better than using the eigenvectors of $\mathbf{S}$ matrix, in the sense of smaller norms of approximation error vectors.

\section{A. Definition and Some Basic Properties}

Let us define an $N \times N$ nearly tridiagonal matrix $\mathbf{T}$ as (8), shown at the bottom of the page. That is, the nonzero entries of $\mathbf{T}$ are

$$
\begin{aligned}
\mathbf{T}(n, n) & =\cos ^{2}(n \pi / N), \quad 0 \leq n \leq(N-1), \\
\mathbf{T}(N-1,0) & =\mathbf{T}(0, N-1)=0.5, \\
\mathbf{T}(n, n+1) & =\mathbf{T}(n+1, n) \\
& =\frac{\cos \frac{n \pi}{N} \cos \frac{(n+1) \pi}{N}}{2 \cos (\pi / N)}, 0 \leq n \leq(N-2) .
\end{aligned}
$$

$$
\mathbf{T}=\left[\begin{array}{cccccc}
1 & 0.5 & 0 & \ldots & 0 & 0.5 \\
0.5 & \left(\cos \frac{\pi}{N}\right)^{2} & \frac{\cos \frac{\pi}{N} \cos \frac{2 \pi}{N}}{2 \cos (\pi / N)} & \ldots & 0 & 0 \\
0 & \frac{\cos \frac{\pi}{N} \cos \frac{2 \pi}{N}}{2 \cos (\pi / N)} & \left(\cos \frac{2 \pi}{N}\right)^{2} & \ldots & 0 & 0 \\
\vdots & \vdots & \vdots & \ddots & \vdots & \vdots \\
0 & 0 & 0 & \ldots & {\left[\cos \frac{(N-2) \pi}{N}\right]^{2}} & \frac{\cos \frac{(N-2) \pi}{N} \cos \frac{(N-1) \pi}{N}}{2 \cos (\pi / N)} \\
0.5 & 0 & 0 & \ldots & \frac{\cos \frac{(N-2) \pi}{N} \cos \frac{(N-1) \pi}{N}}{2 \cos (\pi / N)} & {\left[\cos \frac{(N-1) \pi}{N}\right]^{2}}
\end{array}\right]
$$


Note that except for the two 0.5 entries at the upper-right and lower-left corners, $\mathbf{T}$ is tridiagonal, which is similar to the $\mathbf{S}$ matrix of (6). Thus, we call them nearly tridiagonal. Since $\mathbf{T}$ is real and symmetric, $\mathbf{T}$ has real and orthogonal eigenvectors.

The rationale that we define $\mathbf{T}$ in the form of (8) is described as follows. In [1], Grünbaum introduced an exactly tridiagonal matrix which commutes with the centered discrete Fourier transform matrix of even size. For example, we first consider the case that the sizes of these two matrices are both $4 \times 4$. The $4 \times 4$ centered discrete Fourier transform matrix $\mathbf{F}_{\mathrm{C}}$ and its exactly tridiagonal commuting matrix $\mathbf{T}_{\mathrm{G}}$ defined in [1] are then, respectively

$$
\begin{gathered}
\left(\mathbf{F}_{\mathrm{C}}\right)_{k n}=\frac{1}{2} \cdot e^{j \frac{2 \pi}{4}(k-2)(n-2)}, \quad 1 \leq k, n \leq 4, \\
\mathbf{T}_{\mathrm{G}}=\left[\begin{array}{cccc}
\sin ^{2} \frac{\pi}{4} & \frac{\sin \frac{\pi}{4} \sin \frac{2 \pi}{4}}{2 \cos (\pi / 4)} & 0 & 0 \\
\frac{\sin \frac{\pi}{4} \sin \frac{2 \pi}{4}}{2 \cos (\pi / 4)} & \sin ^{2} \frac{2 \pi}{4} & \frac{\sin \frac{2 \pi}{4} \sin \frac{3 \pi}{4}}{2 \cos (\pi / 4)} & 0 \\
0 & \frac{\sin \frac{2 \pi}{4} \sin \frac{3 \pi}{4}}{2 \cos (\pi / 4)} & \sin ^{2} \frac{3 \pi}{4} & \frac{\sin \frac{3 \pi}{4} \sin \frac{4 \pi}{4}}{2 \cos (\pi / 4)} \\
0 & 0 & \frac{\sin \frac{3 \pi}{4} \sin \frac{4 \pi}{4}}{2 \cos (\pi / 4)} & \sin ^{2} \frac{4 \pi}{4}
\end{array}\right] .
\end{gathered}
$$

Let us define a permutation matrix $\mathbf{P}$ as

$$
\mathbf{P}=\left[\begin{array}{llll}
0 & 1 & 0 & 0 \\
0 & 0 & 1 & 0 \\
0 & 0 & 0 & 1 \\
1 & 0 & 0 & 0
\end{array}\right]
$$

It can be shown that, after performing row and column permutations on $\mathbf{F}_{\mathrm{C}}$, the resultant matrix $\mathbf{P} \mathbf{F}_{\mathrm{C}} \mathbf{P}^{\mathrm{T}}$ is the $4 \times 4$ inverse DFT matrix $\left(\mathbf{F}_{4}\right)^{-1}$. From $\mathbf{F}_{\mathrm{C}} \cdot \mathbf{T}_{\mathrm{G}}=\mathbf{T}_{\mathrm{G}} \cdot \mathbf{F}_{\mathrm{C}}$ and $\mathbf{P}^{\mathrm{T}} \mathbf{P}=\mathbf{I}$, we have that $\mathbf{P T}_{\mathrm{G}} \mathbf{P}^{\mathrm{T}}$ commutes with $\mathbf{P} \mathbf{F}_{\mathrm{C}} \mathbf{P}^{\mathrm{T}}$. Therefore, $\mathbf{P} \mathbf{T}_{\mathrm{G}} \mathbf{P}^{\mathrm{T}}$ commutes with $\left(\mathbf{F}_{4}\right)^{-1}$ and thus also commutes with $\mathbf{F}_{4}$. We conclude that the following matrix is a new commuting matrix of the four-point DFT matrix $\mathbf{F}_{4}$

$$
\begin{aligned}
\mathbf{P T}_{\mathrm{G}} \mathbf{P}^{T} \\
=\left[\begin{array}{cccc}
\sin ^{2} \frac{2 \pi}{4} & \frac{\sin \frac{2 \pi}{4} \sin \frac{3 \pi}{4}}{2 \cos (\pi / 4)} & 0 & \frac{\sin \frac{\pi}{4} \sin \frac{2 \pi}{4}}{2 \cos (\pi / 4)} \\
\frac{\sin \frac{2 \pi}{4} \sin \frac{3 \pi}{4}}{2 \cos (\pi / 4)} & \sin ^{2} \frac{3 \pi}{4} & \frac{\sin \frac{3 \pi}{4} \sin \frac{4 \pi}{4}}{2 \cos (\pi / 4)} & 0 \\
0 & \frac{\sin \frac{3 \pi}{4} \sin \frac{4 \pi}{4}}{2 \cos (\pi / 4)} & \sin ^{2} \frac{4 \pi}{4} & 0 \\
\sin \frac{\pi}{4} \sin \frac{2 \pi}{4} & 0 & 0 & \sin ^{2} \frac{\pi}{4}
\end{array}\right] \\
=\left[\begin{array}{cccc}
2 \cos (\pi / 4) & 0.5 & 0 & 0.5 \\
0.5 & \cos ^{2} \frac{\pi}{4} & \frac{\cos \frac{\pi}{4} \cos \frac{2 \pi}{4}}{2 \cos (\pi / 4)} & 0 \\
0 & \frac{\cos \frac{\pi}{4} \cos \frac{2 \pi}{4}}{2 \cos (\pi / 4)} & \cos ^{2} \frac{2 \pi}{4} & \frac{\cos \frac{2 \pi}{4} \cos \frac{3 \pi}{4}}{2 \cos (\pi / 4)} \\
0.5 & 0 & \frac{\cos \frac{2 \pi}{4} \cos \frac{3 \pi}{4}}{2 \cos (\pi / 4)} & \cos ^{2} \frac{3 \pi}{4}
\end{array}\right] . \quad(13)
\end{aligned}
$$

The above discussion is about the four-point case. We then generalize (13) to the $N$-point case and define the nearly tridiagonal matrix $\mathbf{T}$ in (8). We find that, whenever $N$ is even or odd, $\mathbf{T}$ has the following important property.

Property 1: The $N \times N$ matrix $\mathbf{T}$ in (8) commutes with the $N \times N$ DFT matrix $\mathbf{F}$ defined in (5), i.e., $\mathbf{T F}=\mathbf{F T}$.

Proof: See Appendix I.

To explore the relation of eigenspaces between $\mathbf{F}$ and $\mathbf{T}$, we need the following property.

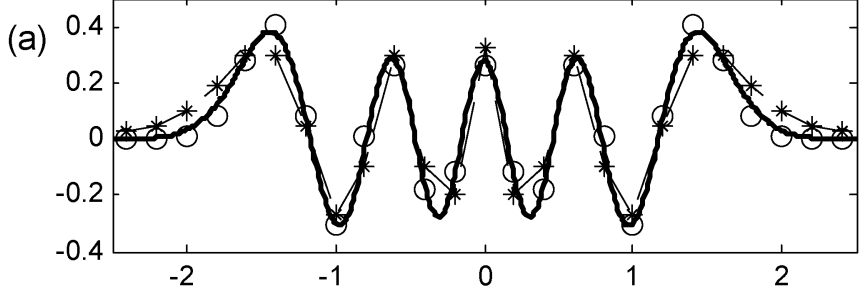

(b)
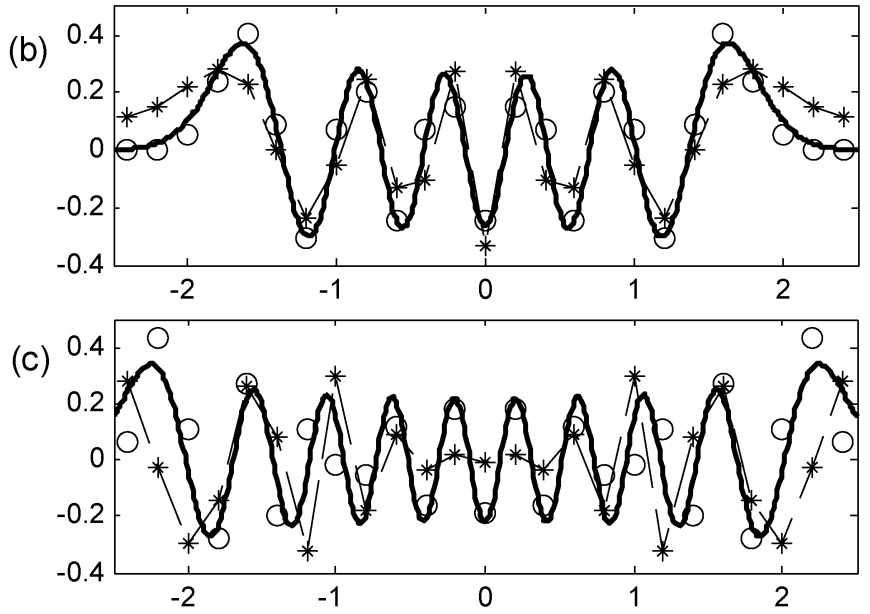

Fig. 1. Continuous HGFs (solid line), the discrete Hermite-Gaussian-like functions based on $\mathbf{S}$ ('*'), and the discrete Hermite-Gaussian-like functions based on $\mathbf{T}$ ("o"), with $N=25$. (a) Eighth order: The error norms of $\mathbf{S}$ and $\mathbf{T}$ are 0.2637 and 0.0959 , respectively. (b) Tenth order: The error norms of $\mathbf{S}$ and $\mathbf{T}$ are 0.4965 and 0.1472 . (c) Eighteenth order: The error norms of $\mathbf{S}$ and $\mathbf{T}$ are 0.9312 and 0.5795 .

Property 2: Let $E_{\lambda}$ be the eigenspace of $\mathbf{T}$ corresponding to eigenvalue $\lambda$, i.e., $E_{\lambda}=\{\mathbf{x} \mid \mathbf{T x}=\lambda \cdot \mathbf{x}\}$. If $\mathbf{x} \in E_{\lambda}$, then $\mathbf{F x} \in E_{\lambda}$.

Proof: Since $\mathbf{x} \in E_{\lambda}, \mathbf{T x}=\lambda \mathbf{x}$. From Property 1 , $\mathbf{T}(\mathbf{F x})=(\mathbf{F T}) \mathbf{x}=\mathbf{F}(\mathbf{T x})=\lambda(\mathbf{F x})$.

From Property 2, we can find the eigenvector set of $\mathbf{F}$ from that of $\mathbf{T}$. Note the following.

1) From Property 2, it can be seen that if $\mathbf{x}$ is the eigenvector of $\mathbf{T}$ corresponding to an eigenvalue with multiplicity 1 , then $\mathbf{X}$ is also an eigenvector of $\mathbf{F}$.

2) If $\mathbf{x}$ is an eigenvector of $\mathbf{T}$ corresponding to an eigenvalue with multiple multiplicities, $\mathbf{x}$ may not be an eigenvector of $\mathbf{F}$.

In Appendix II, we show that the entries of the eigenvectors of $\mathbf{T}$ are solutions of a discrete version of the second-order differential equation of the continuous HGFs [4]. Therefore, the eigenvectors of $\mathbf{T}$ approximate the continuous HGFs. To motivate our further discussions, we perform some computer experiments to show that the differences between the DFT eigenvectors derived from $\mathbf{T}$ and the samples of continuous HGFs are usually smaller than those between the DFT eigenvectors obtained from $\mathbf{S}$ and the samples of continuous HGFs. Its reason is also illustrated in Appendix II.

Computer Experiment 1: Fig. 1(a)-(c) shows the eighth-, tenth-, and eighteenth-order continuous HGFs, the discrete Hermite-Gaussian-like functions based on $\mathbf{S}$, and the discrete Hermite-Gaussian-like functions based on $\mathbf{T}$, with $N=25$. From Fig. 1, we observe that the discrete Hermite-Gaussian-like functions based on $\mathbf{T}$ are closer to the continuous HGFs than those 

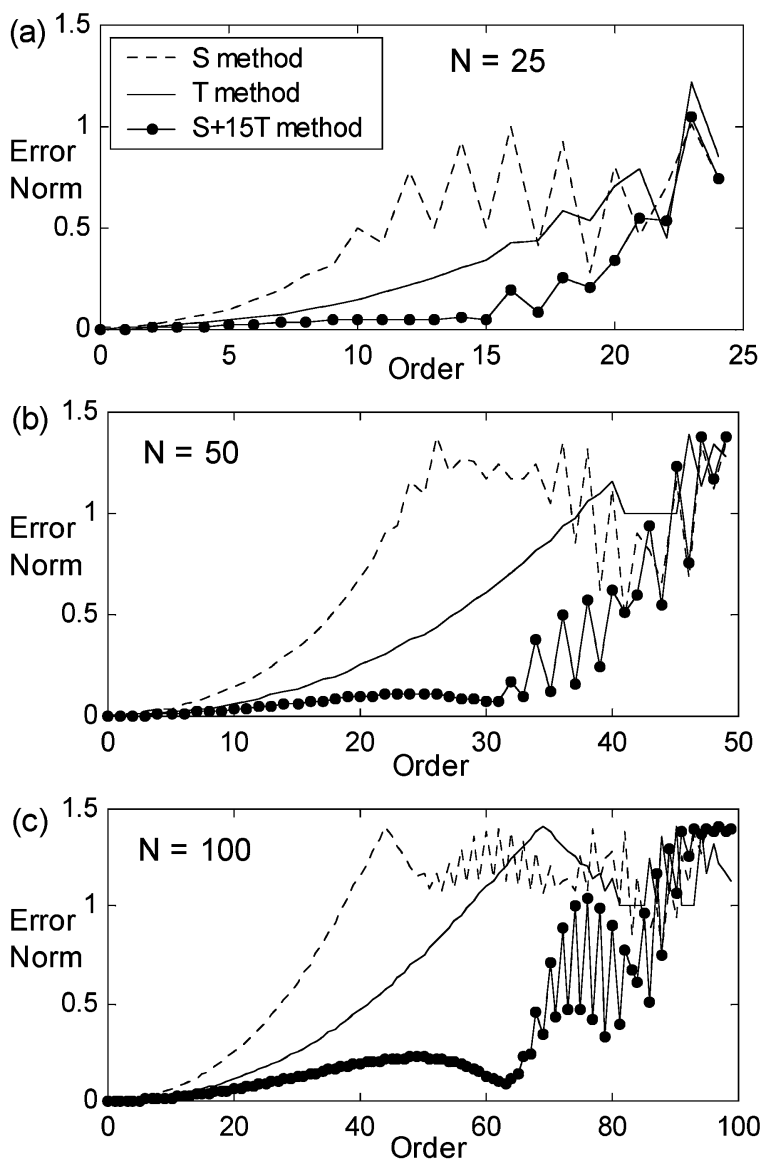

Fig. 2. Error norms of the discrete Hermite-Gaussian-like functions based on $\mathbf{S}(---), \mathbf{T}(-)$, and $\mathbf{S}+15 \mathbf{T}(\longrightarrow \bullet)$ ) of various orders, where (a) $N=25$, (b) $N=50$, and (c) $N=100$.

based on $\mathbf{S}$. The error norms, which are the Euclidean norms of the error vectors between the discrete Hermite-Gaussian-like functions based on $\mathbf{T}$ (or $\mathbf{S}$ ) and samples of the continuous HGFs, are plotted in Fig. 2. The error norms of the discrete Hermite-Gaussian-like functions based on both $\mathbf{S}$ and $\mathbf{T}$ tend to increase for higher order ones because of the aliasing effects. We also show in Fig. 2 the error norms for the discrete Hermite-Gaussian-like functions based on $\mathbf{S}+15 \mathbf{T}$, which will be explained in detail in Section IV.

Fig. 2 shows that the discrete Hermite-Gaussian-like functions based on $\mathbf{T}$ approximate the continuous HGFs with smaller error norms than those based on $\mathbf{S}$ except for the case where the order is very high. We did Matlab experiments for each of $N$ (from $N=15$ to $N=110$ ) and found that when the order is smaller than $k_{0}$, where

$$
\begin{aligned}
& k_{0} \approx 0.77 N-1.25 \quad \text { when } 15 \leq N \leq 60 \\
& k_{0} \approx 0.53 N+9.58 \text { when } 70 \leq N \leq 110
\end{aligned}
$$

the error norm of the discrete Hermite-Gaussian-like function based on $\mathbf{T}$ is smaller than that based on $\mathbf{S}$. When the order is larger than $k_{0}$, sometimes the discrete Hermite-Gaussian-like function based on $\mathbf{T}$ has larger error norm but sometimes its error norm is still less. In fact, for orders larger than $k_{0}$, the discrete Hermite-Gaussian-like functions based on both $\mathbf{S}$ and $\mathbf{T}$ fail to approximate the continuous HGFs well. Since the relation between the discrete Hermite-Gaussian-like function (denoted

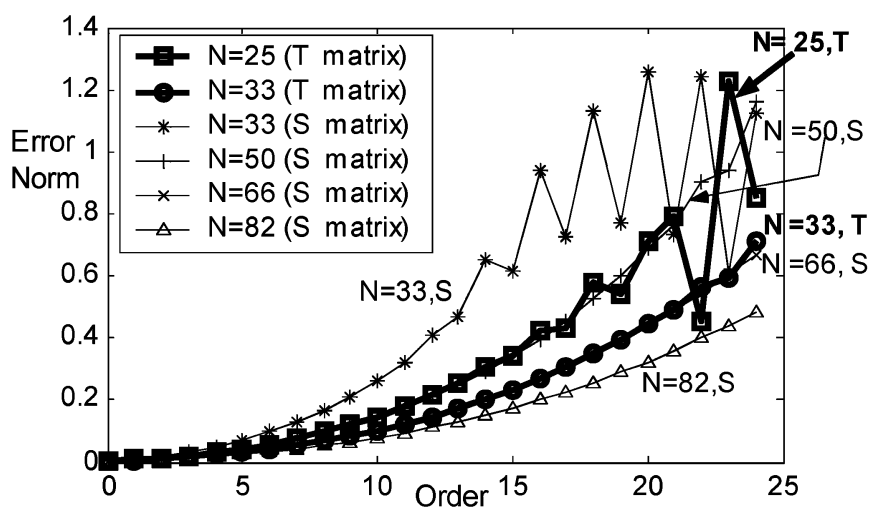

Fig. 3. Error norms of discrete Hermite-Gaussian-like functions based on $\mathbf{S}$ and $\mathbf{T}$ for different $N$.

by $\mathbf{x}$ ) based on $\mathbf{T}$ (or $\mathbf{S}$ ) and the continuous HGF (denoted by $h(t))$ is (proven in Appendix II)

$$
x[n] \approx h(n / \sqrt{N})
$$

and the effect that using $x[n]$ to approximate $h(n / \sqrt{N})$ may get worse when $n / N$ is large (this fact can also be seen from Appendix II), thus if the time support of $h(t)$ is large, it is difficult to use $x[n]$ to approximate $h(t)$ well. Here, we define the time support of the continuous HGF as the threshold $R$ such that $h(t)<0.01$ for $|t| \geq R / 2$. The time support of the continuous HGF grows with its order. This is a possible interpretation as to why we cannot use the discrete Hermite-Gaussian-like function based on $\mathbf{S}$ or $\mathbf{T}$ to approximate it well when the order is high. From our experiments, we find that when the time supports of the continuous HGFs exceed $R$, where

$$
\begin{aligned}
& R \approx 0.4197 \sqrt{N}+3.2859(\text { for } \mathbf{S}) \\
& R \approx 0.5806 \sqrt{N}+3.2847(\text { for } \mathbf{T})
\end{aligned}
$$

the error norms of the discrete Hermite-Gaussian-like functions will be larger than 0.8 .

From these experiments, we expect that, if we define a new DFRFT based on $\mathbf{T}$, the transform outputs will be closer to the samples of the continuous FRT than those of the DFRFT based on $\mathbf{S}$, especially for input signals with their spectra concentrated mostly at low frequencies. Therefore, the performances of the DFRFT based on $\mathbf{T}$ will be similar to the continuous FRT. Many useful properties of the continuous FRT (such as the rotation in the WDF and the property that the shifting operation in the time domain corresponds to the mixture of the shifting and the modulation operations in the DFRFT domain) also apply for the DFRFT based on $\mathbf{T}$.

In Fig. 3, we vary $N$ and compare the error norms of the eigenvectors obtained from $\mathbf{S}$ and $\mathbf{T}$. We find that the error norms of $\mathbf{T}$ with $N$ points correspond to the error norms of $\mathbf{S}$ with $2 N$ points for lower order discrete HGFs. We have known that, when $N$ increases, the DFT eigenvectors obtained from $\mathbf{S}$ and $\mathbf{T}$ will converge to continuous HGFs. From the experiment in Fig. 3, we can conclude that the convergence rate of the eigenvectors derived from $\mathbf{T}$ will be twice of that of the eigenvectors derived from $\mathbf{S}$. This interesting phenomenon will be helpful for the further exploration of the DFT eigenvectors. 


\section{B. Computing DFT Eigenvectors Using Commuting Matrix $\mathbf{T}$}

In [4], with $\mathbf{S}$ defined in (6) being commutative with $\mathbf{F}$, Candan et al. introduced a method to find the common eigenvector set of $\mathbf{S}$ and $\mathbf{F}$. In this subsection, we develop a method to compute eigenvectors of $\mathbf{F}$ completely from those of $\mathbf{T}$.

Property 3: For the $N \times N$ T matrix defined in (8), the transformed matrix

$$
\overline{\mathbf{T}}=\mathbf{U T U}=\left[\begin{array}{cc}
\mathbf{M}_{1} & \mathbf{0} \\
\mathbf{0} & \mathbf{M}_{2}
\end{array}\right]
$$

is a block diagonal matrix, where $\mathbf{M}_{\mathbf{1}}$ and $\mathbf{M}_{\mathbf{2}}$ are two square matrices of sizes $\lfloor N / 2+1\rfloor$ and $\lfloor(N-1) / 2\rfloor$, respectively. $\lfloor x\rfloor$ denotes the largest integer less than or equal to $x$, and $\mathbf{U}$ is the $N \times N$ unitary symmetric matrix defined by [4]

$$
\begin{aligned}
& \mathbf{U}=\frac{1}{\sqrt{2}}\left[\begin{array}{ccc}
\sqrt{2} & \mathbf{0} & \mathbf{0} \\
\mathbf{0} & \mathbf{I}_{\frac{N-1}{2}} & \mathbf{J}_{\frac{N-1}{2}} \\
\mathbf{0} & \mathbf{J}_{\frac{N-1}{2}} & -\mathbf{I}_{\frac{N-1}{2}}
\end{array}\right], \quad \text { if } N \text { is odd } \\
& \mathbf{U}=\frac{1}{\sqrt{2}}\left[\begin{array}{cccc}
\sqrt{2} & \mathbf{0} & 0 & \mathbf{0} \\
\mathbf{0} & \mathbf{I}_{\frac{N}{2}-1} & \mathbf{0} & \mathbf{J}_{\frac{N}{2}-1} \\
0 & \mathbf{0} & \sqrt{2} & \mathbf{0} \\
\mathbf{0} & \mathbf{J}_{\frac{N}{2}-1} & \mathbf{0} & -\mathbf{I}_{\frac{N}{2}-1}
\end{array}\right], \quad \text { if } N \text { is even }
\end{aligned}
$$

where

$$
\mathbf{J}_{q}=\left[\begin{array}{ccc}
\mathbf{0} & & 1 \\
& . & \\
1 & & \mathbf{0}
\end{array}\right]_{q \times q}(\text { i.e., the } q \times q \text { exchange matrix })
$$

Proof: (a) If $N$ is odd

$$
\begin{aligned}
\overline{\mathbf{T}}= & \mathbf{U T U}=\frac{1}{2}\left[\begin{array}{ccc}
\sqrt{2} & \mathbf{0} & \mathbf{0} \\
\mathbf{0} & \mathbf{I} & \mathbf{J} \\
\mathbf{0} & \mathbf{J} & \mathbf{- I}
\end{array}\right] \\
& \times\left[\begin{array}{ccc}
1 & 0.5 \mathbf{e}_{1}^{T} & 0.5 \mathbf{e}_{1}^{T} \mathbf{J} \\
0.5 \mathbf{e}_{1} & \mathbf{T}_{1} & \mathbf{A} \\
0.5 \mathbf{J e}_{1} & \mathbf{J A J} & \mathbf{J T}_{1} \mathbf{J}
\end{array}\right]\left[\begin{array}{ccc}
\sqrt{2} & \mathbf{0} & \mathbf{0} \\
\mathbf{0} & \mathbf{I} & \mathbf{J} \\
\mathbf{0} & \mathbf{J} & -\mathbf{I}
\end{array}\right] \\
= & {\left[\begin{array}{ccc}
1 & \frac{1}{\sqrt{2}} \mathbf{e}_{1}^{T} & \mathbf{0} \\
\frac{1}{\sqrt{2}} \mathbf{e}_{1} & \mathbf{T}_{1}+\mathbf{A J} & \mathbf{0} \\
\mathbf{0} & \mathbf{0} & \mathbf{J T}_{1} \mathbf{J}-\mathbf{J A}
\end{array}\right] } \\
= & {\left[\begin{array}{cc}
\mathbf{M}_{1} & \mathbf{0} \\
\mathbf{0} & \mathbf{M}_{2}
\end{array}\right] }
\end{aligned}
$$

where $\mathbf{e}_{\mathbf{1}}$ is $[1,0,0, \ldots, 0]^{\mathrm{T}}$ of size $(N-1) / 2, \mathbf{T}_{\mathbf{1}}$ is an $(N-$ $1) / 2 \times(N-1) / 2$ submatrix of $\mathbf{T}$, and $\mathbf{A}$ is an $(N-1) / 2 \times$ $(N-1) / 2$ submatrix of $\mathbf{T}$. Entries of $\mathbf{A}$ are zeros except for the entry at the lower-left corner.

(b) If $N$ is even

$$
\begin{aligned}
\overline{\mathbf{T}} & =\mathbf{U T U} \\
& =\frac{1}{2}\left[\begin{array}{cccc}
\sqrt{2} & \mathbf{0} & 0 & \mathbf{0} \\
\mathbf{0} & \mathbf{I} & \mathbf{0} & \mathbf{J} \\
0 & \mathbf{0} & \sqrt{2} & \mathbf{0} \\
\mathbf{0} & \mathbf{J} & \mathbf{0} & \mathbf{- I}
\end{array}\right]\left[\begin{array}{cccc}
1 & 0.5 \mathbf{e}_{1}^{T} & 0 & 0.5 \mathbf{e}_{1}^{T} \mathbf{J} \\
0.5 \mathbf{e}_{1} & \mathbf{T}_{2} & \mathbf{0} & \mathbf{0} \\
0 & \mathbf{0} & 0 & \mathbf{0} \\
0.5 \mathbf{J e}_{1} & \mathbf{0} & \mathbf{0} & \mathbf{J} \mathbf{T}_{2} \mathbf{J}
\end{array}\right]
\end{aligned}
$$

$$
\begin{aligned}
& \times\left[\begin{array}{cccc}
\sqrt{2} & \mathbf{0} & 0 & \mathbf{0} \\
\mathbf{0} & \mathbf{I} & \mathbf{0} & \mathbf{J} \\
0 & \mathbf{0} & \sqrt{2} & \mathbf{0} \\
\mathbf{0} & \mathbf{J} & \mathbf{0} & \mathbf{- I}
\end{array}\right] \\
& =\left[\begin{array}{cccc}
1 & \frac{\sqrt{2}}{2} \mathbf{e}_{1}^{T} & 0 & \mathbf{0} \\
\frac{\sqrt{2}}{2} \mathbf{e}_{1} & \mathbf{T}_{2} & \mathbf{0} & \mathbf{0} \\
0 & \mathbf{0} & 0 & \mathbf{0} \\
\mathbf{0} & \mathbf{0} & \mathbf{0} & \mathbf{J} \mathbf{T}_{2} \mathbf{J}
\end{array}\right]=\left[\begin{array}{cc}
\mathbf{M}_{1} & \mathbf{0} \\
\mathbf{0} & \mathbf{M}_{2}
\end{array}\right]
\end{aligned}
$$

where $\mathbf{e}_{\mathbf{1}}$ is the vector $[1,0,0, \ldots, 0]^{\mathrm{T}}$ of size $(N / 2-1)$ and $\mathbf{T}_{\mathbf{2}}$ is an $(N / 2-1) \times(N / 2-1)$ submatrix of $\mathbf{T}$. The sizes of $\mathbf{M}_{1}$ and $\mathbf{M}_{2}$ are $(N / 2+1) \times(N / 2+1)$ and $(N / 2-1) \times(N / 2-1)$, respectively. Combining (a) and (b), we complete the proof.

From the proof of Property 3, we have that the submatrices $\mathbf{M}_{1}$ and $\mathbf{M}_{2}$ of the transformed matrix $\overline{\mathbf{T}}=\mathbf{U T U}$ are

$$
\begin{aligned}
& \mathbf{M}_{1}=\left[\begin{array}{cc}
1 & \frac{1}{\sqrt{2}} \mathbf{e}_{1}^{T} \\
\frac{1}{\sqrt{2}} \mathbf{e}_{1} & \mathbf{T}_{1}+\mathbf{A} \mathbf{J}
\end{array}\right], \text { if } N \text { is odd } \\
& \mathbf{M}_{1}=\left[\begin{array}{ccc}
1 & \frac{\sqrt{2}}{2} \mathbf{e}_{1}^{T} & 0 \\
\frac{\sqrt{2}}{2} \mathbf{e}_{1} & \mathbf{T}_{2} & \mathbf{0}_{\frac{N}{2}-1} \\
0 & \mathbf{0}_{\frac{N}{2}-1}^{T} & 0
\end{array}\right], \text { if } N \text { is even } \\
& \mathbf{M}_{2}=\mathbf{J}_{\frac{N-1}{2}} \mathbf{T}_{1} \mathbf{J}_{\frac{N-1}{2}}-\mathbf{J}_{\frac{N-1}{2}} \mathbf{A}, \quad \text { if } N \text { is odd } \\
& \mathbf{M}_{2}=\mathbf{J}_{N / 2-1} \mathbf{T}_{2} \mathbf{J}_{N / 2-1}, \quad \text { if } N \text { is even }
\end{aligned}
$$

where $\mathbf{0}_{N / 2-1}$ is the $(N / 2-1) \times 1$ zero vector, and $\mathbf{J}_{q}$ is the exchange matrix of size $q \times q$. We have the following two comments on eigenvalue multiplicities of $\mathbf{M}_{1}$ and $\mathbf{M}_{2}$.

1) If $N$ is odd: From the definition of $\mathbf{T}$ in (8), we see that $\mathbf{T}_{1}$ in (22) is symmetric and exactly tridiagonal with nonzero subdiagonal entries, and $\mathbf{A J}$ is a matrix whose entries are all zero except a nonzero entry at the lower-right corner. From (22), we conclude that $\mathbf{M}_{1}$ is symmetric and exactly tridiagonal with nonzero subdiagonal entries. Similarly, we have that $\mathbf{M}_{2}$ in (23) is also symmetric and exactly tridiagonal with nonzero subdiagonal entries. From [15], we know that any symmetric and exactly tridiagonal matrix with nonzero subdiagonal entries has distinct eigenvalues. Therefore, if $N$ is odd, the diagonal block matrices $\mathbf{M}_{1}$ and $\mathbf{M}_{2}$ of $\overline{\mathbf{T}}=\mathbf{U T U}$ in (17) have distinct eigenvalues.

2) If $N$ is even: From the definition of $\mathbf{T}$ in (8), we can see that $\mathbf{T}_{2}$ in (22) is symmetric and tridiagonal with nonzero subdiagonal entries, thus $\mathbf{M}_{1}$ in (22) is symmetric and exactly tridiagonal. Besides, except that the entries at the last row and last column are all zero, subdiagonal entries of $\mathbf{M}_{1}$ are nonzero. We can see directly from (22) that $\mathbf{M}_{1}$ has two $(N / 2+1) \times 1$ independent eigenvectors corresponding to the zero eigenvalue: $[0,0, \ldots, 0,1]^{T}$ and

$$
\begin{aligned}
& {\left[1,(-1)^{1} \cdot \sqrt{2},(-1)^{2} \cdot \sqrt{2}, \cdots,\right.} \\
& \left.\quad(-1)^{\left(\frac{N}{2}-2\right)} \cdot \sqrt{2},(-1)^{\left(\frac{N}{2}-1\right)} \cdot \sqrt{2}, 0\right]^{T} .
\end{aligned}
$$

Therefore, with the exception that the zero eigenvalue of $\mathbf{M}_{1}$ is of multiplicity two, $\mathbf{M}_{1}$ has distinct eigenvalues. As to the matrix $\mathbf{M}_{2}$ in (23), we can easily see that it is symmetric and tridiagonal with nonzero subdiagonal entries. Thus, $\mathbf{M}_{2}$ has distinct eigenvalues. 
From (17), the relations of eigenvalues and eigenvectors between $\mathbf{T}$ and the diagonal block matrices of $\overline{\mathbf{T}}, \mathbf{M}_{1}$ and $\mathbf{M}_{2}$, can be stated as the following two facts:

1) if $\mathbf{x}_{\mathbf{1}}$ is an eigenvector of $\mathbf{M}_{1}$ corresponding to eigenvalue $\lambda_{1}$, then $\mathbf{y}_{\mathbf{1}}=\mathbf{U} \cdot\left[\mathbf{x}_{\mathbf{1}}^{\mathbf{T}}, \mathbf{0}^{\mathbf{T}}\right]^{\mathbf{T}}$, which is the even extension of $\mathbf{x}_{\mathbf{1}}$, is an eigenvector of $\mathbf{T}$ corresponding to eigenvalue $\lambda_{1}$

2) if $\mathbf{x}_{\mathbf{2}}$ is an eigenvector of $\mathbf{M}_{2}$ corresponding to eigenvalue $\lambda_{2}$, then $\mathbf{y}_{\mathbf{2}}=\mathbf{U} \cdot\left[\mathbf{0}^{\mathbf{T}}, \mathbf{x}_{\mathbf{2}}^{\mathbf{T}}\right]^{\mathbf{T}}$, which is the odd extension of $\mathbf{x}_{2}$, is an eigenvector of $\mathbf{T}$ corresponding to eigenvalue $\lambda_{2}$.

Therefore, we can compute eigenvalues of $\mathbf{T}$ from the union of eigenvalues of $\mathbf{M}_{1}$ and $\mathbf{M}_{\mathbf{2}}$, and compute even and odd eigenvectors of $\mathbf{T}$ from even extensions and odd extensions of eigenvectors of $\mathbf{M}_{\mathbf{1}}$ and $\mathbf{M}_{\mathbf{2}}$, respectively. From previous discussions, $\mathbf{M}_{\mathbf{1}}$ and $\mathbf{M}_{2}$ have distinct eigenvalues with the exception that, when $N$ is even, the zero eigenvalue of $\mathbf{M}_{\mathbf{1}}$ is of multiplicity two.

Property 4: a) If $\mathbf{x}_{\mathbf{1}}$ is an eigenvector, corresponding to any eigenvalue if $N$ is odd or to a nonzero eigenvalue if $N$ is even, of $\mathbf{M}_{\mathbf{1}}$ in (17), then its even extension, $\mathbf{U} \cdot\left[\mathbf{x}_{\mathbf{1}}^{\mathbf{T}}, \mathbf{0}^{\mathbf{T}}\right]^{\mathbf{T}}$, is an eigenvector of $\mathbf{F}$. (b) If $\mathbf{x}_{\mathbf{2}}$ is an eigenvector of $\mathbf{M}_{2}$ in (17), then its odd extension, $\mathbf{U} \cdot\left[\mathbf{0}^{\mathbf{T}}, \mathbf{x}_{\mathbf{2}}^{\mathbf{T}}\right]^{\mathbf{T}}$, is an eigenvector of $\mathbf{F}$.

Proof: a) Assume that $\mathbf{x}_{\mathbf{1}}$ is an eigenvector of $\mathbf{M}_{\mathbf{1}}$ corresponding to eigenvalue $\lambda_{1}$, which is any value if $N$ is odd or is nonzero if $N$ is even. From (17), $\lambda_{1}$ is also an eigenvalue of $\mathbf{T}$. Since we do not know whether $\lambda_{1}$ can be also an eigenvalue of $\mathbf{M}_{\mathbf{2}}$, we divide the discussion into two cases:

a.1) If the multiplicity of $\lambda_{1}$ of $\mathbf{T}$ is $1, \lambda_{1}$ is not an eigenvalue of $\mathbf{M}_{2}$ and thus the eigenspace of $\mathbf{T}$ corresponding to $\lambda_{1}, E_{\lambda 1}$, is of dimension 1. From Property 2, the even extension of $\mathbf{x}_{\mathbf{1}}$ is an eigenvector of $\mathbf{F}$.

a.2) When $\lambda_{1}$ is an eigenvalue of multiplicity one of both $\mathbf{M}_{\mathbf{1}}$ and $\mathbf{M}_{\mathbf{2}}$, if the corresponding eigenvectors of $\lambda_{1}$ of $\mathbf{M}_{\mathbf{1}}$ and $\mathbf{M}_{\mathbf{2}}$ are $\mathbf{x}_{1}$ and $\mathbf{x}_{2}$, respectively, then the eigenspace of $\mathbf{T}$ corresponding to $\lambda_{1}$ is of dimension two and is $E_{\lambda 1}=\operatorname{span}\left(\left\{y_{1}, y_{2}\right\}\right)$ with $\mathbf{y}_{\mathbf{1}}=\mathbf{U} \cdot\left[\mathbf{x}_{\mathbf{1}}^{\mathbf{T}}, \mathbf{0}^{\mathbf{T}}\right]^{\mathbf{T}}$ and $\mathbf{y}_{\mathbf{2}}=\mathbf{U} \cdot\left[\mathbf{0}^{\mathbf{T}}, \mathbf{x}_{\mathbf{2}}^{\mathbf{T}}\right]^{\mathbf{T}}$. From Property 2 , with $\mathbf{y}_{\mathbf{1}}=$ $\mathbf{U} \cdot\left[\mathbf{x}_{\mathbf{1}}^{\mathbf{T}}, \mathbf{0}^{\mathbf{T}}\right]^{\mathbf{T}} \in E_{\lambda 1}$, we have $\mathbf{F} \mathbf{y}_{\mathbf{1}} \in E_{\lambda 1}$. That is

$$
\mathbf{F} \mathbf{y}_{\mathbf{1}}=\beta \cdot \mathbf{y}_{\mathbf{1}}+\gamma \cdot \mathbf{y}_{\mathbf{2}}
$$

with $\mathbf{y}_{1}$ being even and $\mathbf{y}_{2}$ being odd. Since $\mathbf{y}_{1}$ is even, $\mathbf{F y}_{1}$ in (25) is even [5]. Using the fact that any even vector is orthogonal to the odd vector, we have $\gamma=0$. Therefore, $\mathbf{y}_{\mathbf{1}}$ is an eigenvector of $\mathbf{F}$

$$
\mathbf{F y}_{\mathbf{1}}=\beta \cdot \mathbf{y}_{\mathbf{1}}
$$

b) The proof is similar to a). The proof of Property 4 is completed.

From Property 4, we can compute most of the eigenvectors of $\mathbf{F}$ from even extensions and odd extensions of eigenvectors of submatrices $\mathbf{M}_{1}$ and $\mathbf{M}_{2}$ of $\overline{\mathbf{T}}=\mathbf{U T U}$, respectively. But if $N$ is even, zero is an eigenvalue of $\mathbf{M}_{1}$ of multiplicity two and Property 4 does not apply for this case. Thus, if $N$ is even, the even extensions of eigenvectors corresponding to the zero eigenvalue of $\mathbf{M}_{1}$ are not necessarily eigenvectors of $\mathbf{F}$. For $N$ even, we then need to develop a method to compute the eigenvectors of $\mathbf{F}$ in the even subspace spanned by eigenvectors of $\mathbf{T}$ of the zero eigenvalue.

Property 5: If $N$ is even, the two orthogonal eigenvectors of $\mathbf{F}$ in the subspace spanned by even eigenvectors of $\mathbf{T}$ of eigenvalue zero are $[1,-1,1,-1, \ldots, 1,-1]^{\mathrm{T}} \pm \sqrt{N} \mathbf{e}_{\boldsymbol{N} / \mathbf{2}+\mathbf{1}}$, where $\mathbf{e}_{\boldsymbol{N} / \mathbf{2}+\mathbf{1}}$ is the $N$-length column vector with zero entries except a 1 at the $(N / 2+1)^{\text {th }}$ entry.

Proof: It is not difficult to verify from definition of $\mathbf{T}$ in (8) that, if $N$ is even, $[1,-1,1,-1, \ldots, 1,-1]^{\mathrm{T}}$ and $\mathbf{e}_{\boldsymbol{N} / \mathbf{2}+\mathbf{1}}$ are two independent even eigenvectors of $\mathbf{T}$ corresponding to the zero eigenvalue. The eigenspace of $\mathbf{T}$ corresponding to the zero eigenvalue is then $E_{0}=\left\{c_{1} \cdot[1,-1,1,-1, \ldots, 1,-1]^{\mathrm{T}}+c_{2}\right.$. $\left.\mathbf{e}_{\boldsymbol{N} / \mathbf{2}+\mathbf{1}}\right\}$, where $c_{1}$ and $c_{2}$ are any constants. From Property 2 , it is reasonable to assume that $\mathbf{z}=[1,-1,1,-1, \ldots, 1,-1]^{\mathrm{T}} \pm$ $c \cdot \mathbf{e}_{\boldsymbol{N} / \mathbf{2}+\mathbf{1}}$ is an eigenvector of $\mathbf{F}$ in $E_{0}$, with $c$ being a constant. Since even eigenvectors corresponds to eigenvalue \pm 1 for $\mathbf{F}$ [4], we assume $\mathbf{F z}=\lambda \cdot \mathbf{z}= \pm \mathbf{z}$, which results in $c= \pm \sqrt{N}$ if $\lambda= \pm 1$. Therefore, the eigenvector of $\mathbf{F}$ in $E_{0}$ corresponding to eigenvalue $\lambda=1$ is

$$
\mathbf{z}_{\mathbf{1}}=[1,-1,1,-1, \ldots, 1,-1]^{\mathrm{T}}+\sqrt{N} \cdot \mathbf{e}_{\boldsymbol{N} / \mathbf{2}+\mathbf{1}}
$$

and the eigenvector of $\mathbf{F}$ in $E_{0}$ corresponding to eigenvalue $\lambda=$ -1 is

$$
\mathbf{z}_{2}=[1,-1,1,-1, \ldots, 1,-1]^{\mathrm{T}} \cdot \sqrt{N} \cdot \mathbf{e}_{\mathbf{N} / \mathbf{2}+\mathbf{1}} .
$$

It can be easily seen that $\mathbf{z}_{\mathbf{1}}$ and $\mathbf{z}_{\mathbf{2}}$ are orthogonal, and thus the proof is completed.

In this subsection, we develop a method to find the orthogonal common eigenvector set of $\mathbf{F}$ and $\mathbf{T}$ from the eigen-decomposition of $\mathbf{T}$. To define DFRFT based on $\mathbf{T}$, we also need to determine the orders of the discrete Hermite-Gaussian-like functions based on $\mathbf{T}$.

\section{Determining the Hermite-Gaussian Orders of Matrix $\mathbf{T}$ Eigenvectors}

In [4], Candan et al. proposed a rule to assign orders for the Hermite-Gaussian eigenvectors of $\mathbf{S}$ in (6). Similar to the procedures in [4], we determine the orders of the eigenvectors of $\mathbf{T}$ as follows.

We have shown that the diagonal block matrices $\mathbf{M}_{\mathbf{1}}$ and $\mathbf{M}_{2}$ in (17) are both tridiagonal. Therefore, the eigenvector of $\mathbf{M}_{1}$ or $\mathbf{M}_{2}$ of the largest eigenvalue has no zero-crossing, the eigenvector of $\mathbf{M}_{1}$ or $\mathbf{M}_{\mathbf{2}}$ of the second largest eigenvalue has one zero-crossing, etc. [4]. Thus, the eigenvectors of $\mathbf{T}$ obtained from even extensions of the eigenvectors of $\mathbf{M}_{\mathbf{1}}$ has $0,2,4, \cdots, 2 \cdot\lfloor(N / 2)\rfloor$ zero-crossings. The eigenvectors of $\mathbf{T}$ obtained from odd extensions of the eigenvectors of $\mathbf{M}_{\mathbf{2}}$ has $1,3,5, \cdots, 2 \cdot\lfloor((N-3) / 2)\rfloor+1$ zero-crossings. We can then assign the eigenvector of $\mathbf{T}$ with $k$ zero-crossings as the $k^{\text {th }}$-order discrete Hermite-Gaussian-like function. It should be noticed that, if $N$ is even, $\mathbf{T}$ has two even eigenvectors corresponding to the zero eigenvalue. They have the largest and the second largest zero-crossings and should be determined separately from the following.

1) From Property 5, when $N=4 \mathrm{~m}$, since $N / 2+1$ is odd, the eigenvector with the second most zero-crossings is $[1,-1,1,-1, \ldots, 1,-1]^{\mathrm{T}}-\sqrt{N} \mathbf{e}_{\boldsymbol{N} / \mathbf{2}+\mathbf{1}}$, 
and the eigenvector with the most zero-crossings is $[1,-1,1,-1, \ldots, 1,-1]^{\mathrm{T}}+\sqrt{N} \mathbf{e}_{\boldsymbol{N} / \mathbf{2}+\mathbf{1}}$.

2) When $N=4 \mathrm{~m}+2$, since $N / 2+1$ is even, the eigenvector with the second most zero-crossings is $[1,-1,1,-1, \ldots, 1,-1]^{\mathrm{T}}+\sqrt{N} \mathbf{e}_{\boldsymbol{N} / \mathbf{2}+\mathbf{1}}$, and the eigenvector with the most zero-crossings is $[1,-1,1,-1, \ldots, 1,-1]^{\mathrm{T}}-\sqrt{N} \mathbf{e}_{\boldsymbol{N} / \mathbf{2}+\mathbf{1}}$.

\section{LINEAR COMBINATIONS OF MATRICES S AND T}

From previous discussions, the DFT matrix $\mathbf{F}$ has two linearly independent commuting matrices, $\mathbf{S}$ and $\mathbf{T}$, which is defined in (6) and (8), respectively. Since the eigenvectors of both $\mathbf{S}$ and $\mathbf{T}$ are HGF-like, we can expect that eigenvectors of $k_{1} \mathbf{S}+$ $k_{2} \mathbf{T}$ are also discrete Hermite-Gaussian-like functions. Moreover, from our experiments, we observed that the following phenomena always occur:

$$
\begin{array}{ll}
\text { if } x_{\mathrm{S}}[n]<h(n / \sqrt{N}) & \text { then } x_{\mathrm{T}}[n]>h(n / \sqrt{N}) \\
\text { if } x_{\mathrm{S}}[n]>h(n / \sqrt{N}) & \text { then } x_{\mathrm{T}}[n]<h(n / \sqrt{N})
\end{array}
$$

where $\mathbf{x}_{\mathrm{S}}$ and $\mathbf{x}_{\mathrm{T}}$ are the DFT eigenvectors derived from $\mathbf{S}$ and $\mathbf{T}$ and $h(t)$ is the continuous HGF. Thus, we can conjecture that if we combine $\mathbf{S}$ and $\mathbf{T}$ properly, we can obtain the DFT eigenvectors that are very close to $h(n / \sqrt{N})$. From (29), we can conclude that both $k_{1}$ and $k_{2}$ should be positive. Moreover, since, compared with $\mathbf{S}$, the eigenvectors of $\mathbf{T}$ are more similar to the samples of HGFs, $k_{2}$ should be much larger than $k_{1}$. In this section, we show that, with proper choices of $k_{1}$ and $k_{2}$, the DFT eigenvector set derived from $k_{1} \mathbf{S}+k_{2} \mathbf{T}$ can very well approximate the samples of continuous HGFs. The approximation errors are even less than those of the case where we use the DFT eigenvectors derived from $\mathbf{S}$ or $\mathbf{T}$ to approximate the samples of continuous HGFs.

\section{A. New Versions of Discrete Hermite-Gaussian-Like Functions}

Property 6: If $k_{1}$ and $k_{2}$ are any two constants, then $k_{1} \mathbf{S}+$ $k_{2} \mathbf{T}$ commutes with the DFT matrix $\mathbf{F}$, where $\mathbf{S}$ and $\mathbf{T}$ are defined in (6) and (8), respectively. we have

Proof: Using the fact that both $\mathbf{S}$ and $\mathbf{T}$ commute with $\mathbf{F}$,

$$
\begin{aligned}
\left(k_{1} \mathbf{S}+k_{2} \mathbf{T}\right) \cdot \mathbf{F} & =k_{1} \mathbf{S F}+k_{2} \mathbf{T F}=k_{1} \mathbf{F S}+k_{2} \mathbf{F T} \\
& =\mathbf{F} \cdot\left(k_{1} \mathbf{S}+k_{2} \mathbf{T}\right) .
\end{aligned}
$$

From Property 6, we can compute the eigenvectors of DFT matrix $\mathbf{F}$ using $k_{1} \mathbf{S}+k_{2} \mathbf{T}$. Since $k_{1} \mathbf{S}+k_{2} \mathbf{T}$ and $\mathbf{S}+\left(k_{2} / k_{1}\right) \mathbf{T}$ have the same eigenvectors if $k_{1}$ is nonzero, we discuss in the following linear combinations of $\mathbf{S}$ and $\mathbf{T}$ of the form $\mathbf{S}+$ $k \mathbf{T}$ and assume $k \geq 0$. We can easily observe that if $k$ approaches zero, eigenvectors of $\mathbf{S}+k \mathbf{T}$ approaches those of $\mathbf{S}$. On the other hand, if $k \gg 1$, eigenvectors of $\mathbf{S}+k \mathbf{T}$ approaches those of $\mathbf{T}$. We next show through computer experiments that eigenvectors of $\mathbf{S}+k \mathbf{T}$ are new versions of discrete Hermite-Gaussian-like functions and, with appropriate choice of $k$, these new discrete Hermite-Gaussian-like functions approximate samples of the continuous HGFs better than those (a)

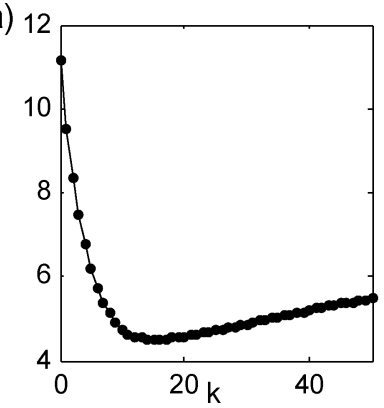

(b) 140

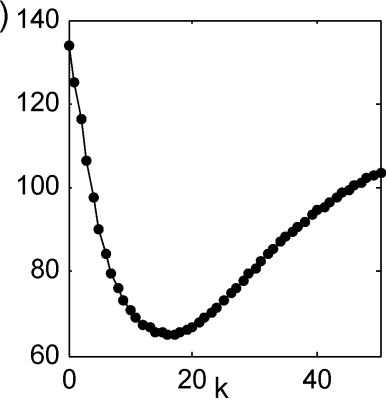

Fig. 4. Total error norms of discrete Hermite-Gaussian-like functions based on $\mathbf{S}+k \mathbf{T}$. (a) $N=25$. (b) $N=145$.

obtained from both $\mathbf{S}$ and $\mathbf{T}$, in the sense that most of these new discrete Hermite-Gaussian-like functions have smaller norms of approximation error vectors.

Computer Experiment 2: To determine the optimal choice of $k$, in the sense of the minimal total error norm, we first compute the eigenvectors of $\mathbf{S}+k \mathbf{T}$, which are new versions of discrete Hermite-Gaussian-like functions. All of the resulting $N$ eigenvectors are compared with samples of the continuous HGFs of the corresponding orders and the total error norms are calculated. For $N=25$ and $N=145$, the total error norms are plotted versus various values of $k$ (from $k=0$ to $k=50$ with spacing 1) in Fig. 4(a) and (b). From these results and other experiments for different values of $N$ (up to 145), we find that the optimal $k$ in the sense of the minimal total error norm is approximately 15 . For $N=25,50$, and 100, in Fig. 2, we plot the error norms of discrete Hermite-Gaussian-like functions of various orders based on $\mathbf{S}, \mathbf{T}$, and $\mathbf{S}+k \mathbf{T}$ with $k=15$. It is obvious that discrete Hermite-Gaussian-like functions based on $\mathbf{S}+15 \mathbf{T}$ outperform those based on both $\mathbf{S}$ and $\mathbf{T}$.

\section{B. Computing DFT Eigenvectors from Those of $\mathbf{S}+k \mathbf{T}$}

Property 7: For the $N \times N$ S matrix defined in (6)

$$
\overline{\mathbf{S}}=\mathbf{U S U}=\left[\begin{array}{cc}
\mathbf{M}_{3} & \mathbf{0} \\
\mathbf{0} & \mathbf{M}_{4}
\end{array}\right]
$$

is a block diagonal matrix [4], where $\mathbf{U}$ is the $N \times N$ unitary matrix defined in (18).

Proof: a) If $N$ is odd, similar to the proof a) of Property 3, we have

$$
\begin{aligned}
\overline{\mathbf{S}} & =\mathbf{U} \cdot\left[\begin{array}{ccc}
2 & \mathbf{e}_{1}^{T} & \mathbf{e}_{1}^{T} \mathbf{J} \\
\mathbf{e}_{1} & \mathbf{S}_{1} & \mathbf{B} \\
\mathbf{J e}_{1} & \mathbf{J B J} & \mathbf{J} \mathbf{S}_{1} \mathbf{J}
\end{array}\right] \cdot \mathbf{U} \\
& =\left[\begin{array}{ccc}
2 & \sqrt{2} \mathbf{e}_{1}^{T} & \mathbf{0} \\
\sqrt{2} \mathbf{e}_{1} & \mathbf{S}_{1}+\mathbf{B J} & \mathbf{0} \\
\mathbf{0} & \mathbf{0} & \mathbf{J} \mathbf{S}_{1} \mathbf{J}-\mathbf{J B}
\end{array}\right]
\end{aligned}
$$

where $\mathbf{e}_{\mathbf{1}}$ is $[1,0,0, \ldots, 0]^{\mathrm{T}}$ of size $(N-1) / 2, \mathbf{S}_{\mathbf{1}}$ is an $(N-$ 1) $/ 2 \times(N-1) / 2$ submatrix of $\mathbf{S}$, and $\mathbf{B}$ is an $(N-1) / 2 \times$ $(N-1) / 2$ submatrix of $\mathbf{S}$. The entries of $\mathbf{B}$ are zeros except a 1 at the lower-left corner. 
b) If $N$ is even, similar to the proof b) of Property 3, we have

$$
\begin{aligned}
\overline{\mathbf{S}} & =\mathbf{U} \cdot\left[\begin{array}{cccc}
2 & \mathbf{e}_{1}^{T} & 0 & \mathbf{e}_{1}^{T} \mathbf{J} \\
\mathbf{e}_{1} & \mathbf{S}_{2} & \mathbf{J} \mathbf{e}_{1} & \mathbf{0} \\
0 & \mathbf{e}_{1}^{T} \mathbf{J} & -2 & \mathbf{e}_{1}^{T} \\
\mathbf{J e}_{1} & \mathbf{0} & \mathbf{e}_{1} & \mathbf{J S}_{2} \mathbf{J}
\end{array}\right] \cdot \mathbf{U} \\
& =\left[\begin{array}{cccc}
2 & \sqrt{2} \mathbf{e}_{1}^{T} & 0 & \mathbf{0} \\
\sqrt{2} \mathbf{e}_{1} & \mathbf{S}_{2} & \sqrt{2} \mathbf{J e}_{\mathbf{1}} & \mathbf{0} \\
0 & \sqrt{2} \mathbf{e}_{1}^{T} \mathbf{J} & -2 & \mathbf{0} \\
\mathbf{0} & \mathbf{0} & \mathbf{0} & \mathbf{J S}_{2} \mathbf{J}
\end{array}\right]
\end{aligned}
$$

with $\mathbf{e}_{\mathbf{1}}$ being the vector $[1,0,0, \ldots, 0]^{\mathrm{T}}$ of size $N / 2-1$ and $\mathbf{S}_{\mathbf{2}}$ being the $(N / 2-1) \times(N / 2-1)$ submatrix of $\mathbf{S}$. Combining (a) and (b), we complete the proof.

From the proof of Property 7, we conclude that

$$
\begin{aligned}
& \mathbf{M}_{3}=\left[\begin{array}{cc}
2 & \sqrt{2} \mathbf{e}_{1}^{T} \\
\sqrt{2} \mathbf{e}_{1} & \mathbf{S}_{1}+\mathbf{B J}
\end{array}\right], \quad \text { if } N \text { is odd } \\
& \mathbf{M}_{3}=\left[\begin{array}{ccc}
2 & \sqrt{2} \mathbf{e}_{1}^{T} & 0 \\
\sqrt{2} \mathbf{e}_{1} & \mathbf{S}_{2} & \sqrt{2} \mathbf{J} \mathbf{e}_{1} \\
0 & \sqrt{2} \mathbf{e}_{1}^{T} \mathbf{J} & -2
\end{array}\right], \quad \text { if } N \text { is even } \\
& \mathbf{M}_{4}=\mathbf{J}_{\frac{N-1}{2}} \mathbf{S}_{1} \mathbf{J}_{\frac{N-1}{2}}-\mathbf{J}_{\frac{N-1}{2}} \mathbf{B}, \quad \text { if } N \text { is odd } \\
& \mathbf{M}_{4}=\mathbf{J}_{\frac{N}{2}-1} \mathbf{S}_{2} \mathbf{J}_{\frac{N}{2}-1}, \quad \text { if } N \text { is even. }
\end{aligned}
$$

It is easy to see that $\mathbf{S}+k \mathbf{T}$ is nearly tridiagonal, in the sense that $\mathbf{S}+k \mathbf{T}$ is tridiagonal except the two nonzero entries at the upper-right and lower-left corners. From (17) and (31), we have

$$
\begin{aligned}
\mathbf{U}(\mathbf{S}+k \mathbf{T}) \mathbf{U} & =\mathbf{U S U}+k \mathbf{U T U}=\overline{\mathbf{S}}+k \overline{\mathbf{T}} \\
& =\left[\begin{array}{cc}
\mathbf{M}_{3}+k \mathbf{M}_{1} & \mathbf{0} \\
\mathbf{0} & \mathbf{M}_{4}+k \mathbf{M}_{2}
\end{array}\right]
\end{aligned}
$$

Therefore, we have the following comments.

1) $\mathbf{S}+k \mathbf{T}$ can be transformed to a matrix of block diagonal form from (36).

2) From (22), (23), (34), and (35), $\mathbf{M}_{\mathbf{1}}, \mathbf{M}_{\mathbf{2}}, \mathbf{M}_{\mathbf{3}}$, and $\mathbf{M}_{\mathbf{4}}$ are all symmetric tridiagonal and have positive subdiagonal entries, except that the last subdiagonal entry of $\mathbf{M}_{\mathbf{1}}$ is zero if $N$ is even. Therefore, if $k$ is nonnegative, the diagonal submatrices $\mathbf{M}_{\mathbf{3}}+k \mathbf{M}_{\mathbf{1}}$ and $\mathbf{M}_{\mathbf{4}}+k \mathbf{M}_{\mathbf{2}}$ are both symmetric and tridiagonal with nonzero subdiagonal entries. Thus, both $\mathbf{M}_{\mathbf{3}}+k \mathbf{M}_{\mathbf{1}}$ and $\mathbf{M}_{\mathbf{4}}+k \mathbf{M}_{\mathbf{2}}$ have distinct eigenvalues.

3) Similar to the proof of Property 4 and from the fact that $\mathbf{S}+k \mathbf{T}$ commutes with $\mathbf{F}$, we have that the even extensions of eigenvectors of $\mathbf{M}_{3}+k \mathbf{M}_{\mathbf{1}}$ and the odd extensions of the eigenvectors of $\mathbf{M}_{\mathbf{4}}+k \mathbf{M}_{\mathbf{2}}$ are eigenvectors of $\mathbf{F}$. We can therefore compute all of the eigenvectors of $\mathbf{F}$ from the eigenvectors of $\mathbf{M}_{3}+k \mathbf{M}_{\mathbf{1}}$ and $\mathbf{M}_{4}+k \mathbf{M}_{2}$. The orders of the discrete Hermite-Gaussian-like functions based on $\mathbf{S}+k \mathbf{T}$ can be determined similarly using the procedure similar to that proposed in [4].

\section{DisCRETE FraCtional Fourier TRANSFORM BASED ON T OR $\mathbf{S}+k \mathbf{T}$ AND ITS APPLICATIONS}

From the results in previous sections, we know that we can use the matrix $\mathbf{T}$ or $\mathbf{S}+k \mathbf{T}$ to derive the eigenvectors of $\mathbf{F}$ and the resultant eigenvectors can approximate the continuous HGFs better than those obtained from $\mathbf{S}$, in the sense of smaller (a)

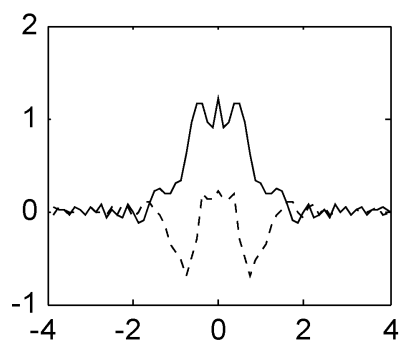

(c)
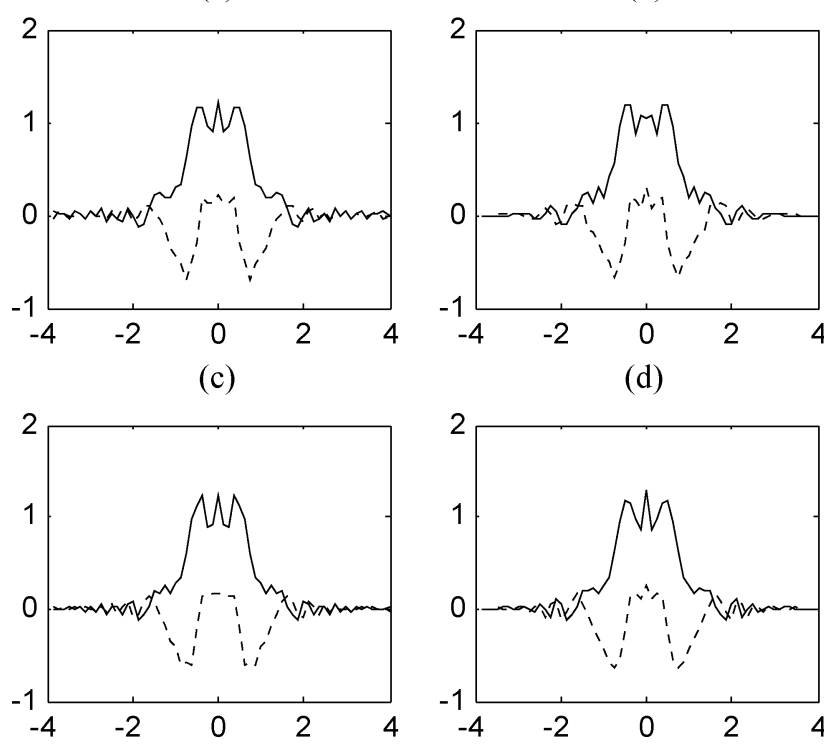

(d)

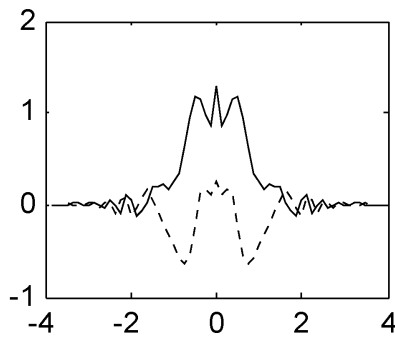

Fig. 5. Comparing the transform results of the continuous FRT and the DFRFTs based on $\mathbf{S}, \mathbf{T}$, and $\mathbf{S}+15 \mathbf{T}$ for a rectangular function (real parts: solid lines, imaginary parts: dashes, order $a=0.25$ ). (a) Continuous FRT $(a=0.25)$; (b) DFRFT based on S (RMSE $=0.0913)$; (c) DFRFT based on $\mathbf{T}(\mathrm{RMSE}=0.0647)$; (d) DFRFT based on $\mathbf{S}+15 \mathbf{T}(\mathrm{RMSE}=0.0526)$.

norms of approximation error vectors. Since the obtained DFT eigenvectors is very close to the eigenfunctions of the continuous Fourier transform, it is possible to define the DFRFT whose performance is very similar to the continuous FRT. The DFRFT based on $\mathbf{T}$ (or $\mathbf{S}+k \mathbf{T}$ ) is

$$
\begin{aligned}
\mathbf{F}_{\mathbf{T}}^{a} & =\mathbf{G D}^{a} \mathbf{G}^{T} \\
& = \begin{cases}\sum_{r=0}^{N-1} e^{-j \pi r a / 2} \mathbf{g}_{r} \mathbf{g}_{r}^{T}, & \text { for } N \text { odd } \\
\sum_{r=0}^{N-2} e^{-j \pi r a / 2} \mathbf{g}_{r} \mathbf{g}_{r}^{T} & \\
+e^{-j \pi N a / 2} \mathbf{g}_{N} \mathbf{g}_{N}^{T}, & \text { for } N \text { even }\end{cases}
\end{aligned}
$$

where $\mathbf{G}=\left[\mathrm{g}_{0}\left|\mathrm{~g}_{1}\right| \ldots\left|\mathrm{g}_{N-2}\right| \mathrm{g}_{N-1}\right]$ for odd $N, \mathbf{G}=$ $\left[\mathrm{g}_{0}\left|\mathrm{~g}_{1}\right| \ldots\left|\mathrm{g}_{N-2}\right| \mathrm{g}_{\boldsymbol{N}}\right]$ for even $N$, and $\mathrm{g}_{r}$ is the $r^{\text {th }}$-order normalized DFT eigenvectors computed from $\mathbf{T}$ (or $\mathbf{S}+k \mathbf{T}$ ).

Computer Experiment 3: To compare the performance, we first compute the continuous FRT and the DFRFTs based on $\mathbf{S}$, $\mathbf{T}$ and $\mathbf{S}+15 \mathbf{T}$ of the following rectangular function

$$
x(t)=1 \text { when }|t| \leq 17 / 16, \quad x(t)=0 \text { elsewhere. }
$$

The continuous FRT is computed by numerical integration of the definition of FRT in (1). The DFRFTs based on $\mathbf{S}, \mathbf{T}$, and $\mathbf{S}+15 \mathbf{T}$ for the samples of $x(t)$ in (38) are computed with $N=64$ and sampling interval is $1 / 8$. The transform results are plotted in Fig. 5 with order $a=0.25$. We find that the transform results of the DFRFTs based on $\mathbf{T}$ and $\mathbf{S}+15 \mathbf{T}$ are more similar to those of the continuous FRT. Their root-mean-square errors (RMSEs) are less than that of the DFRFT based on $\mathbf{S}$.

In Fig. 6, we change the transform order from 0.1 to 1 and compare the RMSE. We find that, for any order $a$, the DFRFTs based on $\mathbf{T}$ and $\mathbf{S}+15 \mathbf{T}$ can well approximate the continuous 


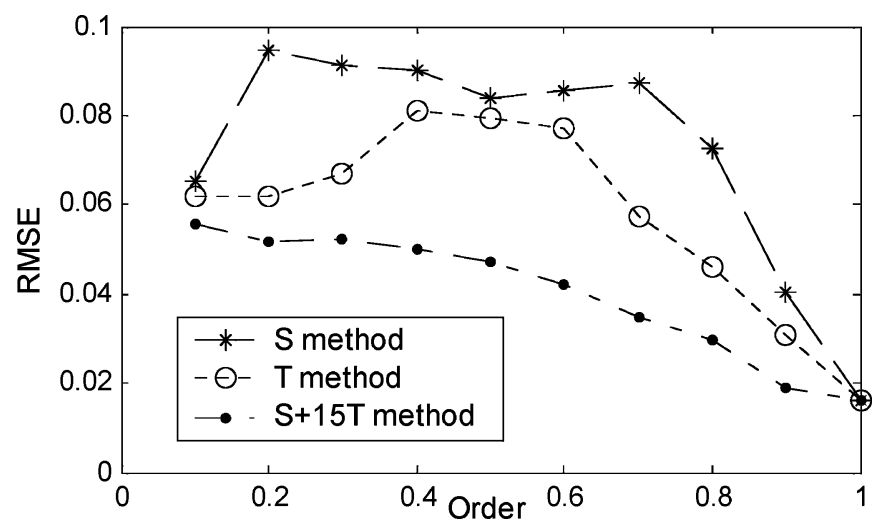

Fig. 6. RMSE of DFRFTs based on $\mathbf{S}, \mathbf{T}$ and $\mathbf{S}+15 \mathbf{T}$ of the samples of the rectangular function in (38).

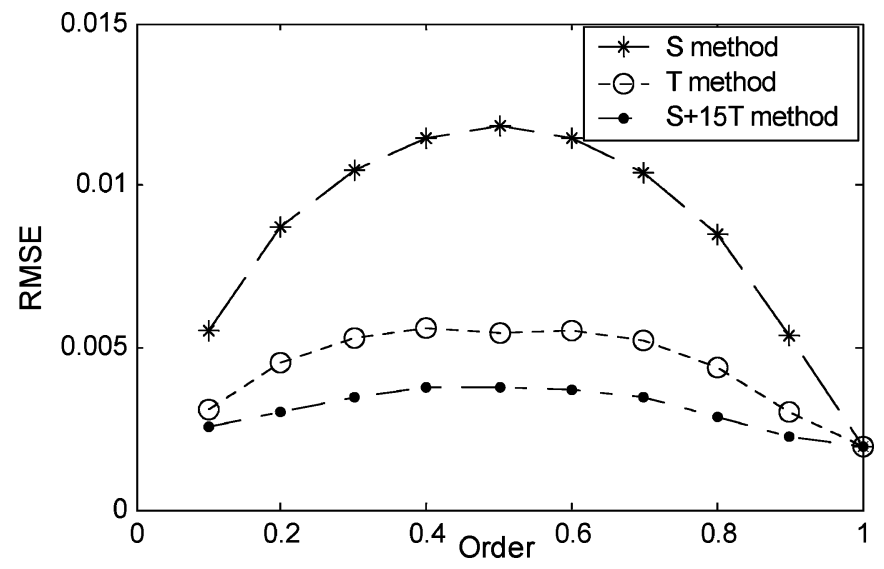

Fig. 7. RMSE of DFRFTs based on $\mathbf{S}, \mathbf{T}$ and $\mathbf{S}+15 \mathbf{T}$ of the samples of the triangular function in (39).

FRT and the RMSEs are less than those of the cases where we apply the DFRFT based on $\mathbf{S}$. The experiment in Fig. 7 is similar to that in Fig. 6, except that the input is a triangular function

$$
x(t)=1-|t| / 2 \text { when }|t| \leq 2, \quad x(t)=0 \text { elsewhere. }
$$

Since the performances of the DFRFTs based on $\mathbf{T}$ and $\mathbf{S}+$ $k \mathbf{T}$ are very close to those of the continuous FRT, the applications of the continuous FRT can also be treated as the applications of the DFRFTs based on $\mathbf{T}$ and $\mathbf{S}+k \mathbf{T}$. For example, it is known that, after doing the continuous FRT of order $a$, the WDF is rotated clockwise by the angle of $a \pi / 2$ [9], [16]-[18]:

$W_{F_{a}}(u, v)=W_{f}(u \cos \alpha-v \sin \alpha, u \sin \alpha+v \cos \alpha)$

where $\alpha=a \pi / 2$.

$W_{f}(u, v)$ means the WDF of $f(u)$

$$
W_{f}(u, v)=\int_{-\infty}^{\infty} f(u+\tau / 2) \cdot f^{*}(u-\tau / 2) \cdot e^{-j 2 \pi v \tau} \cdot d \tau
$$

and $W_{F a}(u, v)$ means the WDF of $F_{a}(u)=\operatorname{FRT}[f(t)]$. In addition

$W_{g}(u, v)=W_{f}(u-k, v-h) \quad$ if $g(t)=\exp (j 2 \pi h) f(t-k)$. (a)

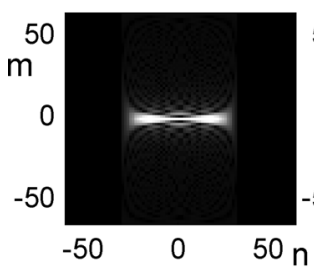

(d)

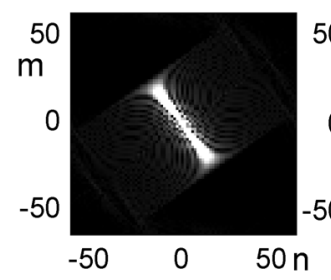

(b)

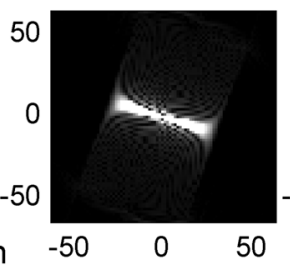

(e)

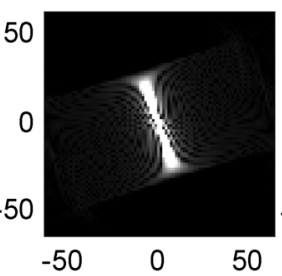

(c)

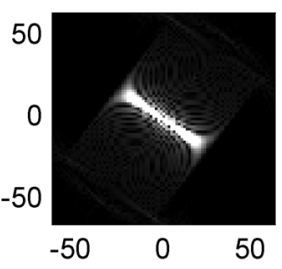

(f)

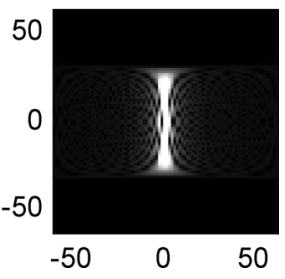

Fig. 8. DWDF of $F_{a}[n]$ where $F_{a}[n]$ is the DFRFT based on $\mathbf{S}+15 \mathbf{T}$ for a rectangular function. (a) $a=0$; (b) $a=0.2$; (c) $a=0.4$; (d) $a=0.6$; (e) $a=0.8$; and (f) $a=1$.

Due to (40) and (42), we can use the continuous FRT together with space-translation and modulation for generalized signal multiplexing and space-frequency slot adaptation [17], [19].

Similarly, we can also use the DFRFT based on $\mathbf{T}$ or $\mathbf{S}+k \mathbf{T}$ for fractional multiplexing and space-frequency slot adaptation, since the properties in (40) and (42) also apply for the DFRFT based on $\mathbf{T}$ or $\mathbf{S}+k \mathbf{T}$. The discrete form of the Wigner distribution function (DWDF) is

$$
W_{f}[n, m]=N^{-1 / 2} \sum_{r} f[n-r] f^{*}[n+r] e^{-j 4 \pi m r / N} .
$$

If $W_{F a}(n, m)$ is the DWDF of $F_{a}[n]$ where $F_{a}[n]$ is the DFRFT of $f[n]$, then it can be shown that

$$
\begin{array}{r}
W_{F_{a}}[n, m] \approx W_{f}[n \cos \alpha-m \sin \alpha, n \sin \alpha+m \cos \alpha] \\
\text { where } \alpha=a \pi / 2 .
\end{array}
$$

In Fig. 8 , we show an example. The input $f[n]$ is a rectangular function:

$f[n]=1 \quad$ when $-32 \leq n \leq 32, \quad f[n]=0$ otherwise. $(45)$ Then we do the 256-point DFRFTs based on $\mathbf{S}+15 \mathrm{~T}$ of orders $0,0.2,0.4,0.6,0.8$, and 1 for $f[n]$ and compute the DWDF. The results are plotted in Fig. 8(a)-(f). We use the gray-level to show the magnitude of the DWDF. From the results, it can be seen that, after doing the DFRFT of order $a$, the DWDF is rotated clockwise by the angle of $a \pi / 2$, as the continuous case.

In Fig. 9, we give an example that uses the DFRFT based on $\mathbf{S}+15 \mathbf{T}$ to do space-frequency slot adaptation (i.e., making the space-frequency distribution of a signal suit for some specific region). Here, the input function $f[n]$ is a chirp-modulated Gaussian function. Its DWDF is plotted in Fig. 9(a):

$$
f[n]=n \cdot \exp \left(-\pi n^{2} / 512\right) \exp \left(-j \pi \cot (0.35 \pi) n^{2} / 256\right) .
$$

We want to multiplex $f[n]$ such that its energy is concentrated on the region of

$$
n \in[10,30], \quad m \in[20,60] \text {. }
$$




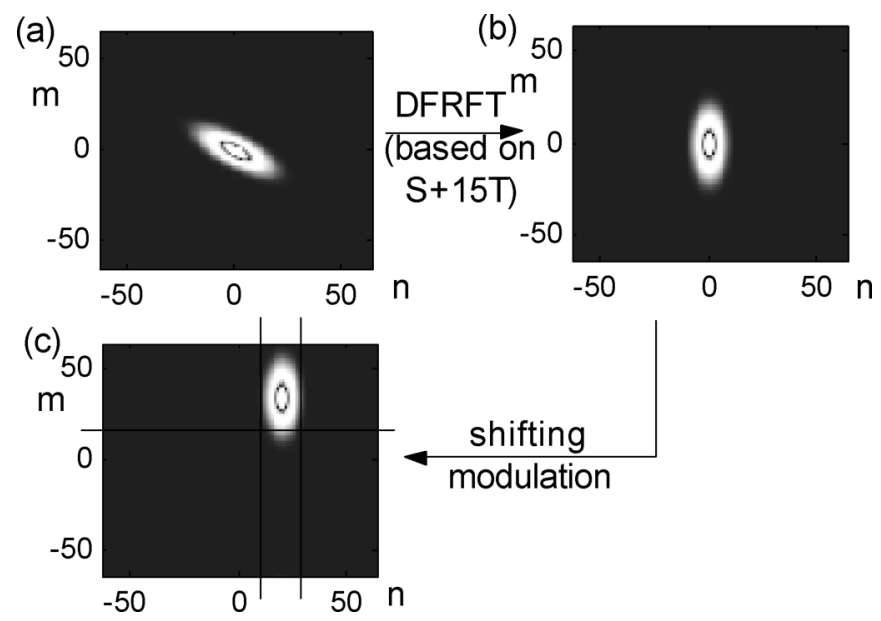

Fig. 9. Doing time-frequency slot adaptation for $f[n]$ such that the energy is concentrated on the rectangular region of $n \in[10,30]$ and $m \in[20,60]$.

First, we do the DFRFT for $f[n]$ to rotate $W_{f}[n, m]$ and make it align with $m$-axis. We first compute the principal direction $\left[d_{1}, d_{2}\right]^{\mathrm{T}}$, which can be determined from the eigenvector corresponding to the larger eigenvalue of the moment covariance matrix $\mathrm{M}$ :

$$
\begin{aligned}
\mathbf{M} & =\left[\begin{array}{ll}
m_{1,1} & m_{1,2} \\
m_{1,2} & m_{2,2}
\end{array}\right] \\
m_{11} & =\sum_{m} \sum_{n} n^{2}\left|W_{f}[n, m]\right| \\
m_{22} & =\sum_{m} \sum_{n} m^{2}\left|W_{f}[n, m]\right| \\
m_{12} & =\sum_{m} \sum_{n} n m\left|W_{f}[n, m]\right| .
\end{aligned}
$$

After some calculation, we find that the principal axis is

$$
\left[d_{1}, d_{2}\right]=[-0.8466,0.5322]
$$

Since

$$
\tan ^{-1}\left(d_{2} / d_{1}\right)=-0.5612=-0.3572(\pi / 2)
$$

thus, from (44), to make the DWDF align with $m$ axis, we should do the DFRFT of order $a$ where

$$
a=-0.3572-(-1)=0.6428 \text {. }
$$

After doing the DFRFT of order 0.6428, the DWDF, is aligned with $m$ axis, as in Fig. 9(b). Then, to shift the DWDF into the desired time-frequency slot, we do space-translation and frequency modulation for the result of the DFRFT (denoted by $\left.F_{0.6428}[n]\right)$

$$
y[n]=\exp (j 2 \pi \cdot 40 n / N) \cdot F_{0.6428}[n-20] .
$$

After doing it, the energy of the DWDF is concentrated on the slot of $n \in[10,30]$ and $m \in[20,60]$, as in Fig. 9(c). Using the DFRFT based on $\mathbf{T}$ or $\mathbf{S}+k \mathbf{T}$ together with space translation and modulation, we can do time-frequency slot adaptation and hence do fractional multiplexing.
Moreover, since the partially space-invariant property is also preserved for the DFRFTs based $\mathbf{T}$ and $\mathbf{S}+k \mathbf{T}$, therefore, as the case of the continuous FRT [9], [20], we can also use them for space-variant pattern recognition. This application is described in detail in our recent paper [24].

In addition to the above applications, other applications of the continuous FRT, such as filter design [11], [12], asymmetric edge detection [22], and matching pursuit [23] are also potential applications of the DFRFTs based on $\mathbf{T}$ and $\mathbf{S}+k \mathbf{T}$.

\section{CONCLUSION}

In this paper, we proposed a new nearly tridiagonal commuting matrix of the DFT. Its eigenvectors were found to be new discrete Hermite-Gaussian-like functions. We showed that most of the eigenvectors of the proposed nearly tridiagonal matrix well approximates the continuous HGFs. The approximation error is smaller than that of the case where we use the eigenvectors derived from $\mathrm{S}$ matrix to approximate continuous HGFs. We also provided rigorous discussions on the properties of eigenvectors and eigenvalues of the new nearly tridiagonal matrix, and gave a method to compute the DFT eigenvectors completely from those of the new nearly tridiagonal matrix. Furthermore, by properly combining two nearly tridiagonal matrices, a new set of commuting matrices, whose DFT eigenvectors are even more similar to the continuous HGFs, were also obtained. Finally, based on these new nearly tridiagonal matrices, new versions of the DFRFT were defined, and their applications were illustrated.

\section{APPENDIX I}

MAtrix T COMMUtes With The DFT MATRIX F (i.e., $\mathbf{T F}=\mathbf{F T}$ )

1) The $(m, n)^{\text {th }}$ entry of $2 \sqrt{N} \cos (\pi / N) \cdot(\mathbf{F T})[1]$ is

$$
\begin{aligned}
(\sqrt{N} \mathbf{F T})_{m, n} \cdot 2 \cos \frac{\pi}{N} \\
=\cos \frac{(n-2) \pi}{N} \cos \frac{(n-1) \pi}{N} e^{-j \frac{2 \pi}{N}(m-1)(n-2)} \\
\quad+2 \cos \frac{\pi}{N}\left(\cos \frac{(n-1) \pi}{N}\right)^{2} e^{-j \frac{2 \pi}{N}(m-1)(n-1)} \\
\quad+\cos \frac{(n-1) \pi}{N} \cos \frac{n \pi}{N} e^{-j \frac{2 \pi}{N}(m-1) n} \\
=e^{-j \frac{2 \pi}{N}(m-1)(n-1)} \cos \frac{(n-1) \pi}{N} \\
\quad \cdot\left[\cos \frac{(n-2) \pi}{N} e^{-j \frac{2 \pi}{N}(m-1)(-1)}+\cos \frac{n \pi}{N} e^{-j \frac{2 \pi}{N}(m-1)(+1)}\right. \\
\left.\quad+2 \cos \frac{\pi}{N} \cos \frac{(n-1) \pi}{N}\right]
\end{aligned}
$$

where

$$
\begin{aligned}
\cos & \frac{(n-2) \pi}{N} e^{-j \frac{2 \pi}{N}(m-1)(-1)}+\cos \frac{n \pi}{N} e^{-j \frac{2 \pi}{N}(m-1)(+1)} \\
= & \cos \frac{2 \pi(m-1)}{N} \cdot 2 \cos \frac{(n-1) \pi}{N} \cos \frac{\pi}{N} \\
& +j \sin \frac{2 \pi(m-1)}{N} \cdot 2 \sin \frac{(n-1) \pi}{N} \sin \frac{\pi}{N}
\end{aligned}
$$


Substituting (A3) into (A2), we obtain

$$
\begin{aligned}
2 \sqrt{N} & \cos \frac{\pi}{N} \cdot(\mathbf{F T})_{m, n} \\
= & {\left[\left(\cos \frac{2 \pi(m-1)}{N}+1\right) \cdot 2 \cos \frac{(n-1) \pi}{N} \cos \frac{\pi}{N}\right.} \\
& \left.+j \sin \frac{2 \pi(m-1)}{N} \cdot 2 \sin \frac{(n-1) \pi}{N} \sin \frac{\pi}{N}\right] \\
& \cdot e^{-j \frac{2 \pi}{N}(m-1)(n-1)} \cos \frac{(n-1) \pi}{N} \\
= & e^{-j \frac{2 \pi}{N}(m-1)(n-1)} \cos ^{2}\left(\frac{\pi(n-1)}{N}\right) \cos \frac{\pi}{N} \\
\cdot & {\left[4 \cos ^{2}\left(\frac{\pi(m-1)}{N}\right) \cos ^{N} \sin \frac{\pi}{N}\right] }
\end{aligned}
$$

2) Similarly, we can see that the $(m, n)^{\text {th }}$ entry of $2 \sqrt{N} \cos (\pi / N) \cdot(\mathbf{T F})$ is

$$
\begin{aligned}
2 \sqrt{N} & \cos \frac{\pi}{N} \cdot(\mathbf{T F})_{m, n} \\
= & \cos \frac{(m-2) \pi}{N} \cos \frac{(m-1) \pi}{N} e^{-j \frac{2 \pi}{N}(n-1)(m-2)} \\
& +2 \cos \frac{\pi}{N}\left(\cos \frac{(m-1) \pi}{N}\right)^{2} e^{-j \frac{2 \pi}{N}(n-1)(m-1)} \\
& +\cos \frac{(m-1) \pi}{N} \cos \frac{m \pi}{N} e^{-j \frac{2 \pi}{N}(n-1) m} .
\end{aligned}
$$

3) Comparing (A5) with (A1), we find that (A5) can be obtained by interchanging $m$ and $n$ in (A1). Since interchanging $m$ and $n$ in (A4) does not change its value, we prove that $(\mathbf{F T})_{\mathbf{m}, \mathbf{n}}=(\mathbf{T F})_{\mathbf{m}, \mathbf{n}}$.

\section{APPENDIX II}

EIGENVECTORS OF T ARE DisCRETE HERMITE-GAUSSIAN-LiKE FUNCTIONS AND THE APPROXIMATION ERROR IS SMALLER

\section{THAN THAT OF THE EIGENVECTORS OF $\mathbf{S}$ MATRIX}

The second-order differential equation for the continuous Hermite-Gaussian function (HGF) $h(t)$ is

$$
\begin{aligned}
& \frac{d^{2}}{d t^{2}} h(t)-4 \pi^{2} t^{2} h(t)=\tau \cdot h(t), \\
& \pi=-2 \pi(2 k+1), \quad k \text { is the order of } h(t) .
\end{aligned}
$$

The central concept of our proof is that, instead of using $\Delta=$ $1 / \sqrt{N}$, we use $\Delta=1 /(r \sqrt{N})$ ( $r$ is any positive integer) to approximate the second-order differentiation in (A6) more accurately, as follows:

$$
\frac{d^{2}}{d t^{2}} h(t) \approx \frac{h(t-\Delta)-2 h(t)+h(t+\Delta)}{\Delta^{2}} \text { where } \Delta=1 /(r \sqrt{N}) .
$$

It is known that the relation between the HGF and the HermiteGaussian-like discrete Fourier transform (DFT) eigenvector is (the case of $\mathbf{S}$ matrix has been proved in [13] and the case of $\mathbf{T}$ matrix will be shown in (A22)-(A26))

$$
x[n] \approx h(n / \sqrt{N}) \quad \text { (i.e., } t=n / \sqrt{N} \text { and } n=t \sqrt{N})
$$

Although for the relation between $t$ and $n$, the interval is $1 / \sqrt{N}$, however, to approximate the second-order differentiation in (A7) more accurately, it is proper to choose an even smaller $\Delta$ instead of $1 / \sqrt{N}$ (i.e., $r>1$ ). The value of $r$ should be as large as possible to make $\Delta$ very small. Then we apply (A6), (A7) and obtain a recursive relation for $h_{n-1 / r}, h_{n}$, and $h_{n+1 / r}$, where

$$
h_{n}=h(n / \sqrt{N})
$$

We then do linear combination to convert it into a recursive relation for $h_{n-1}, h_{n}$, and $h_{n+1}$ (the detail of conversion can be seen from (A10)-(A20) that is an example of $r=3$ ). Then, we compare the result with the difference equations corresponding to $\mathbf{T}$ and $\mathbf{S}$. We can use whose difference equations is closer to the relation among $h_{n-1}, h_{n}$, and $h_{n+1}$ to conclude whether the eigenvectors of $\mathbf{T}$ or of $\mathbf{S}$ can approximate the continuous HGF with smaller approximation error.

We show an example that uses $r=3$, i.e., $\Delta=1 / 3 \sqrt{N}$. From (A6) and (A7)

$$
\begin{aligned}
& h_{n-1 / 3}-c \cdot h_{n}+h_{n+1 / 3} \approx \rho \cdot h_{n}, \\
& \quad \text { where } c=2+4 \pi^{2} n^{2} /\left(9 N^{2}\right), \quad \rho=\tau /(9 N) .
\end{aligned}
$$

We can replace $n$ by $n-2 / 3, n-1 / 3, n+1 / 3$, and $n+2 / 3$ and obtain

$$
\begin{aligned}
& \text { (II) } h_{n-1}-a \cdot h_{n-2 / 3}+h_{n-1 / 3} \approx \rho \cdot h_{n-2 / 3} \\
& \text { (III) } h_{n-2 / 3}-b \cdot h_{n-1 / 3}+h_{n} \approx \rho \cdot h_{n-1 / 3} \\
& \text { (IV) } h_{n}-d \cdot h_{n+1 / 3}+h_{n+2 / 3} \approx \rho \cdot h_{n+1 / 3} \\
& \text { (V) } h_{n+1 / 3}-e \cdot h_{n+2 / 3}+h_{n+1} \approx \rho \cdot h_{n+2 / 3} \\
& a=2+4 \pi^{2}(n-2 / 3)^{2} /\left(9 N^{2}\right) \\
& b=2+4 \pi^{2}(n-1 / 3)^{2} /\left(9 N^{2}\right) \\
& c=2+4 \pi^{2} n^{2} /\left(9 N^{2}\right) \\
& d=2+4 \pi^{2}(n+1 / 3)^{2} /\left(9 N^{2}\right) \\
& e=2+4 \pi^{2}(n+2 / 3)^{2} /\left(9 N^{2}\right), \quad \rho=\tau /(9 N) .
\end{aligned}
$$

In (A10) and (A11), there are seven terms, $h_{n-1}, h_{n-2 / 3}$, $h_{n-1 / 3}, h_{n}, h_{n+1 / 3}, h_{n+2 / 3}$, and $h_{n+1}$. To compare with $\mathbf{S}$ and $\mathbf{T}$, we should do linear combinations for (A10) and (A11) to eliminate the terms of $h_{n-2 / 3}, h_{n-1 / 3}, h_{n+1 / 3}$, and $h_{n+2 / 3}$ (i.e., preserve only the terms of $h_{n}$ and $h_{n \pm 1}$.) It can be done by

$$
\begin{aligned}
& (\mathrm{II}) \times 1+(\mathrm{III}) \times a+(\mathrm{I}) \times(a b-1)+(\mathrm{IV}) \times(a b e-e) /(d e-1) \\
& +(\mathrm{V}) \times(a b-1) /(d e-1) .
\end{aligned}
$$

After some calculation, we obtain

$$
\begin{aligned}
& h_{n-1}-\frac{a+c+e-a d e-c d e-a b c-a b e+a b c d e}{d e-1} h_{n} \\
& +\frac{a b-1}{d e-1} h_{n+1} \\
& \approx \rho\left[h_{n-2 / 3}+a h_{n-1 / 3}+(a b-1) h_{n}\right. \\
& \left.\quad+\frac{a b e-e}{d e-1} h_{n+1 / 3}+\frac{a b-1}{d e-1} h_{n+2 / 3}\right] .
\end{aligned}
$$

Then we try to simplify the right side of (A14). From (A11), since $h_{n-2 / 3} \approx(b+\rho) h_{n-1 / 3}-h_{n}$ and $h_{n+2 / 3} \approx$ 
$(d+\rho) h_{n+1 / 3}-h_{n}$, we can eliminate the terms of $h_{n-2 / 3}$ and $h_{n+2 / 3}$. Moreover, if the order $k$ of the HGF is not large, $\rho=\tau /(9 N)=-2 \pi(2 k+1) /(9 N)$ will be small. Thus, the right side of (A14) becomes

$$
\begin{gathered}
\rho\left[(a+b) h_{n-1 / 3}+\left(a b-2-\frac{a b-1}{d e-1}\right) h_{n}\right. \\
\left.+\frac{a b e-e+a b d-d}{d e-1} h_{n+1 / 3}\right] \\
=\delta+\left(\frac{a+b}{2}+\frac{a b e-e+a b d-d}{2(d e-1)}\right) \rho\left(h_{n-1 / 3}+h_{n+1 / 3}\right) \\
+\left(a b-2-\frac{a b-1}{d e-1}\right) \rho h_{n}
\end{gathered}
$$

where $\delta=(((a+b) / 2)-((a b e-e+a b d-d) /(2(d e-$ 1)))) $\rho\left(h_{n-1 / 3}-h_{n+1 / 3}\right)$. From (A11), $h_{n-1 / 3}+h_{n+1 / 3} \approx$ $(c+\rho) h_{n}$. Thus, (from (A14) and (A15))

$$
\begin{gathered}
h_{n-1}-\frac{a+c+e-a d e-c d e-a b c-a b e+a b c d e}{d e-1} h_{n} \\
+\frac{a b-1}{d e-1} h_{n+1} \\
=\delta+\left(\frac{a b c e-c e+a b c d-c d-2 a b+2}{2(d e-1)}\right. \\
\left.+\frac{a c+b c+2 a b}{2}-2\right) \rho h_{n} \\
\text { (from (A14) and (A15)). }
\end{gathered}
$$

Then, we apply (A12) to express the coefficients of $h_{n}, h_{n+1}$, and $\rho h_{n}$ in terms of $n$ and $N$. It requires very complicated computations. Here we show only the case of $(a b-1) /(d e-1)$ in detail.

$$
\begin{aligned}
a b-1= & \left(2+\frac{4 \pi^{2}(n-2 / 3)^{2}}{9 N^{2}}\right)\left(2+\frac{4 \pi^{2}(n-1 / 3)^{2}}{9 N^{2}}\right)-1 \\
\approx & 3+\frac{\pi^{2}\left(16 n^{2}-16 n+40 / 9\right)}{9 N^{2}}, \\
d e-1= & \left(2+\frac{4 \pi^{2}(n+1 / 3)^{2}}{9 N^{2}}\right)\left(2+\frac{4 \pi^{2}(n+2 / 3)^{2}}{9 N^{2}}\right)-1 \\
\approx & 3+\frac{\pi^{2}\left(16 n^{2}+16 n+40 / 9\right)}{9 N^{2}}, \quad \text { (A17) } \\
\frac{a b-1}{d e-1} \approx & \frac{1+\pi^{2}\left(16 n^{2}-16 n+40 / 9\right) /\left(27 N^{2}\right)}{1+\pi^{2}\left(16 n^{2}+16 n+40 / 9\right) /\left(27 N^{2}\right)} \\
\approx & {\left[1+\frac{\pi^{2}\left(16 n^{2}-16 n+40 / 9\right)}{27 N^{2}}\right] } \\
& \times\left[1-\frac{\pi^{2}\left(16 n^{2}+16 n+40 / 9\right)}{27 N^{2}}\right] \\
\approx & 1+\frac{\pi^{2}\left(16 n^{2}-16 n+40 / 9\right)}{27 N^{2}} \\
& -\frac{\pi^{2}\left(16 n^{2}+16 n+40 / 9\right)}{27 N^{2}} \\
= & 1-\frac{32 \pi^{2} n}{27 N^{2}} .
\end{aligned}
$$

\section{Similarly}

$$
\begin{aligned}
& \frac{-a-c-e+a d e+c d e+a b c+a b e-a b c d e}{d e-1} \\
& \approx-2+\frac{\pi^{2}\left(-108 n^{2}+32 n-16\right)}{27 N^{2}}, \\
& \frac{a c+b c+2 a b}{2}+\frac{a b c e-c e+a b c d-c d-2 a b+2}{2(d e-1)}-2 \\
& \approx 9+\frac{\pi^{2}\left(16 n^{2}-16 n+80 / 27\right)}{3 N^{2}} .
\end{aligned}
$$

Therefore, (A16) and hence (A6) can be approximated by

$$
\begin{aligned}
& h_{n-1}+\left(-2+\frac{\pi^{2}\left(-4 n^{2}+32 n / 27-16 / 27\right)}{N^{2}}\right) h_{n} \\
& +\left(1-\frac{32 \pi^{2} n}{27 N^{2}}\right) h_{n+1} \\
& \quad \approx \delta+\frac{\tau}{N}\left(1+\frac{\pi^{2}\left(16 n^{2}-16 n+80 / 27\right)}{27 N^{2}}\right) h_{n} .
\end{aligned}
$$

Now, we have approximated (A6) by a recursive relation among $h_{n-1}, h_{n}$, and $h_{n+1}$. We compare it with $\mathbf{T}$ matrix and $\mathbf{S}$ matrix. From [13], it is known that, if $\mathbf{y}$ is an eigenvector of $\mathbf{S}$ with eigenvalue $\eta$, then $(\mathbf{S}-4 \mathbf{I}) \mathbf{y}=(\eta-4) \mathbf{y}$ and it can be approximated as (using the fact that $\eta \approx 4-2 \pi(2 k+1)) / N=4+\tau / N$ )

$$
y[n-1]+\left(-2-\frac{4 \pi^{2} n^{2}}{N^{2}}\right) y[n]+y[n+1] \approx \frac{\tau}{N} y[n] .
$$

For $\mathbf{T}$ matrix, let $\mathbf{x}$ be an eigenvector of $\mathbf{T}$ with eigenvalue $\lambda$. From (8), $(2 \mathbf{T}-4 \mathbf{I}) \mathbf{x}$ is

$$
\begin{gathered}
\frac{\cos \frac{(n-1) \pi}{N} \cos \frac{n \pi}{N}}{\cos (\pi / N)} x[n-1]+\left[2\left(\cos \frac{n \pi}{N}\right)^{2}-4\right] x[n]+ \\
\frac{\cos \frac{n \pi}{N} \cos \frac{(n+1) \pi}{N}}{\cos (\pi / N)} x[n+1]=(2 \lambda-4) x[n] .
\end{gathered}
$$

Assume that $N$ is large and $n \ll N$. We can apply the Taylor series for cosine and secant functions, $\cos \phi \approx 1-\phi^{2} / 2$ and $\sec \phi \approx 1+\phi^{2} / 2$. Then, in (A22), the coefficient function of $x[n-1]$ becomes

$$
\begin{aligned}
& \cos \frac{(n-1) \pi}{N} \cos \frac{n \pi}{N} \sec \frac{\pi}{N} \\
& \approx\left(1-\frac{(n-1)^{2} \pi^{2}}{2 N^{2}}\right)\left(1-\frac{n^{2} \pi^{2}}{2 N^{2}}\right)\left(1+\frac{\pi^{2}}{2 N^{2}}\right) \\
& \approx 1-\frac{\left[(n-1)^{2}+n^{2}-1\right] \pi^{2}}{2 N^{2}}=1-\frac{\left(n^{2}-n\right) \pi^{2}}{N^{2}} \\
& 2 \cos ^{2} \frac{n \pi}{N}-4=\cos \frac{2 n \pi}{N}-3 \approx-2-2 \frac{n^{2} \pi^{2}}{N^{2}} \\
& \cos \frac{n \pi}{N} \cos \frac{(n+1) \pi}{N} \sec \frac{\pi}{N} \approx 1-\frac{\left(n^{2}+n\right) \pi^{2}}{N^{2}} .
\end{aligned}
$$

Therefore, (A22) becomes

$$
\begin{aligned}
& \left(1-\frac{\left(n^{2}-n\right) \pi^{2}}{N^{2}}\right) x[n-1]+\left(-2-2 \frac{n^{2} \pi^{2}}{N^{2}}\right) x[n] \\
& +\left(1-\frac{\left(n^{2}+n\right) \pi^{2}}{N^{2}}\right) x[n+1] \\
& \quad \approx(2 \lambda-4) x[n],
\end{aligned}
$$




$$
\begin{aligned}
& x[n-1]+\left(-2-\frac{\pi^{2}\left(4 n^{2}-2 n\right)}{N^{2}}\right) x[n] \\
& +\left(1-\frac{2 \pi^{2} n}{N^{2}}\right) x[n+1] \\
& \quad \approx(2 \lambda-4)\left(1+\frac{\left(n^{2}-n\right) \pi^{2}}{N^{2}}\right) x[n] .
\end{aligned}
$$

In (A23) we apply the fact that $\left[1-\left(n^{2}-n\right) \pi^{2} / N^{2}\right]^{-1} \approx$ $1+\left(n^{2}-n\right) \pi^{2} / N^{2}$ when $N \gg n$. It is not difficult to see that, when $N \gg n,(\mathrm{~A} 25)$ approximates (A20), where

$$
x[n] \approx h(n / \sqrt{N}), \quad \lambda \approx 2+\tau / 2 N=2-\pi(2 k+1)) / N .
$$

Thus, the eigenvector of $\mathbf{T}$ is close to the samples of the continuous HGF and their eigenvalues also have a close relation. Then, we compare (A25) and (A21) with (A20) to conclude whether the eigenvectors of $\mathbf{T}$ or those of $\mathbf{S}$ will be more similar to the continuous HGF. We find the following:

(I) coefficient differences between $\mathbf{S}$ (see (A21)) and the recursive formula for HGFs (see (A20)):

$$
\begin{aligned}
& \text { 2nd term : } \frac{32}{27} \frac{\pi^{2}}{N^{2}} n-\frac{16 \pi^{2}}{27 N^{2}}, \quad 3 \text { rd term }: \frac{32}{27} \frac{\pi^{2}}{N^{2}} n, \\
& \text { right-sided term }: \frac{\pi^{2}\left(16 n^{2}-16 n+80 / 27\right)}{27 N^{2}}
\end{aligned}
$$

(II) coefficient differences between $\mathbf{T}$ (see (A25)) and the recursive formula for HGFs (see (A20)):

2nd term : $\frac{22}{27} \frac{\pi^{2}}{N^{2}} n+\frac{16 \pi^{2}}{27 N^{2}}, \quad 3$ rd term $: \frac{22}{27} \frac{\pi^{2}}{N^{2}} n$,

right-sided term : $\frac{\pi^{2}\left(11 n^{2}-11 n-80 / 27\right)}{27 N^{2}}$.

From (A27) and (A28), it can be seen that the coefficient differences ratios for all the three terms are

16( for $\mathbf{S}$ ) : 11( for $\mathbf{T}$ ) (i.e., 1.4545:1),

when $N$ is large.

That is, the rows of $\mathbf{T}$ are 16/11 times closer to the discrete form of the differential equation of the continuous HGF in (A6) than the rows of $\mathbf{S}$ when choosing $\Delta=1 /(3 \sqrt{N})$.

If in (A12) we choose

$$
\Delta=1 /(4 \sqrt{N}) \quad(r=4)
$$

using the similar process from (A10)-(A29), we find that the coefficient difference ratio becomes

$$
5(\text { for } \mathbf{S}): 3(\text { for } \mathbf{T}) \quad \text { (i.e., } 1.6: 1) \text {. }
$$

When $r=5$, it becomes 16 (for $\mathbf{S}$ ):9 (for $\mathbf{T}$ ) (i.e., 1.778:1). When $r=6$, it becomes 35 (for $\mathbf{S}$ ): 19 (for $\mathbf{T}$ ) (i.e., 1.842:1). In general, when choosing $\Delta=1 /(r \sqrt{N})$, the coefficient difference ratio is

$$
2 r^{3}-2 r(\text { for } \mathbf{S}): r^{3}+2 r(\text { for } \mathbf{T}) .
$$

When $r \rightarrow \infty$, it becomes

$$
2(\text { for } \mathbf{S}): 1(\text { for } \mathbf{T}) \text {. }
$$

That is, the matrix $\mathbf{T}$ is two times closer to the differential equation in (A6) than $\mathbf{S}$ matrix if in (A7) we use a very small $\Delta$ to approximate the second-order differentiation. Therefore, the eigenvectors of $\mathbf{T}$ can approximate the continuous HGF with less error norm.

\section{REFERENCES}

[1] F. A. Grünbaum, "The eigenvectors of the discrete Fourier transform: A version of the Hermite functions," J. Math. Anal. Appl., vol. 88, no. 2, pp. 355-363, 1982.

[2] S. C. Pei and M. H. Yeh, "Improved discrete fractional Fourier transform," Opt. Lett., vol. 22, pp. 1047-1049, 1997.

[3] S. C. Pei, C. C. Tseng, M. H. Yeh, and J. J. Shyu, "Discrete fractional Hartley and Fourier transforms," IEEE Trans. Circuits Syst. II, Analog Digit. Signal Process., vol. 45, pp. 665-675, 1998.

[4] C. Candan, M. A. Kutay, and H. M. Ozaktas, "The discrete fractional Fourier transform," IEEE Trans. Signal Process., vol. 48, no. 5, pp. 1329-1337, May 2000.

[5] S. C. Pei, M. H. Yeh, and C. C. Tseng, "Discrete fractional Fourier transform based on orthogonal projections," IEEE Trans. Signal Process., vol. 47, no. 5, pp. 1335-1348, May 1999.

[6] S. C. Pei and J. J. Ding, "Eigenfunctions of the offset Fourier, fractional Fourier, and linear canonical transforms," J. Opt. Soc. Amer. A, Opt. Image Sci., vol. 20, no. 3, pp. 522-532, Mar. 2003.

[7] C. C. Tseng, "Eigenvalues and eigenvectors of generalized DFT, generalized DHT, DCT-IV and DST-IV matrices," IEEE Trans. Signal Process., vol. 50, no. 4, pp. 866-877, Apr. 2002.

[8] L. B. Almeida, "The fractional Fourier transform and time-frequency representations," IEEE Trans. Signal Process., vol. 42, no. 11, pp. 3084-3091, Nov. 1994.

[9] H. M. Ozaktas, Z. Zalevsky, and M. A. Kutay, The Fractional Fourier Transform With Applications in Optics and Signal Processing. New York: Wiley, 2000.

[10] V. Namias, "The fractional order Fourier transform and its application to quantum mechanics," J. Inst. Math. Appl., vol. 25, pp. 241-265, 1980.

[11] M. A. Kutay, H. M. Ozaktas, L. Onural, and O. Arikan, "Optimal fil tering in fractional Fourier domains," in Proc. IEEE Int. Conf. Acoustics, Speech, Signal Processing (ICASSP), 1995, vol. 2, pp. 937-941.

[12] M. A. Kutay, H. M. Ozaktas, O. Arikan, and L. Onural, "Optimal filtering in fractional Fourier domains," IEEE Trans. Signal Process., vol. 45, pp. 1129-1143, Jul. 1997.

[13] B. W. Dickinson and K. Steiglitz, "Eigenvectors and functions of the discrete Fourier transform," IEEE Trans. Acoust., Speech, Signal Process., vol. ASSP-30, no. 1, pp. 25-31, Jan. 1982.

[14] J. H. McClellan and T. W. Parks, "Eigenvalue and eigenvector decomposition of the discrete Fourier transform," IEEE Trans. Audio. Electroacoust., vol. AU-20, pp. 66-74, 1972.

[15] J. H. Wilkinson, The Algebraic Eigenvalue Problem. Oxford, U.K.: Oxford, 1988.

[16] A. W. Lohmann, "Image rotation, Wigner rotation, and the fractional Fourier transform," J. Opt. Soc. Amer. A, Opt. Image Sci., vol. 10, no. 10, pp. 2181-2186, Oct. 1993.

[17] H. M. Ozaktas, B. Barshan, D. Mendlovic, and L. Onural, "Convolution, filtering, and multiplexing in fractional Fourier domains and their rotation to chirp and wavelet transform," J. Opt. Soc. Amer. A, Opt. Image Sci., vol. 11, no. 2, pp. 547-559, Feb. 1994

[18] S. C. Pei and J. J. Ding, "Relations between the fractional operations and the Wigner distribution, ambiguity function," IEEE Trans. Signal Process., vol. 49, no. 8, pp. 1638-1655, Aug. 2001.

[19] C. Mendlovic and A. W. Lohmann, "Space-bandwidth product adaption and its application to superresolution: Fundamentals," J. Opt. Soc. Amer. A, Opt. Image Sci., vol. 14, no. 3, pp. 558-562, Mar. 1997. 
[20] W. Lohmann, Z. Zalevsky, and D. Mendlovic, "Synthesis of pattern recognition filters for fractional Fourier processing," Opt. Commun., vol. 128, pp. 199-204, Jul. 1996.

[21] B. Santhanam and J. G. Vargas-Rubio, "On the Grünbaum commutor based discrete fractional Fourier transform," in Proc IEEE Int. Conf. Acoustics, Speech, Signal Processing (ICASSP), Montreal, QC, Canada, May 17-21, 2004, vol. II, pp. 641-644.

[22] A. W. Lohmann, D. Mendlovic, and Z. Zalevsky, "Fractional Hilbert transform," Opt. Lett., vol. 21, no. 4, pp. 281-283, Feb. 1996.

[23] A. Bultan, "A four-parameter atomic decomposition of chirplets," IEEE Trans. Signal Process., vol. 47, no. 3, pp. 731-745, Mar. 1999.

[24] S.-C. Pei, W.-L. Hsue, and J.-J. Ding, "Discrete fractional Fourier transform based on new nearly tridiagonal commuting matrices," in Proc. IEEE Int. Conf. Acoustics, Speech, Signal Processing (ICASSP), Philadelphia, PA, Mar. 18-23, 2005, vol. IV, pp. 385-388.

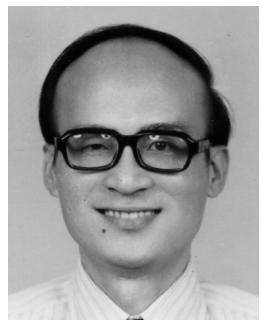

Soo-Chang Pei (SM'89-F'00) was born in Soo-Auo, Taiwan, R.O.C., in 1949. He received B.S.E.E. degree from National Taiwan University, Taipei, Taiwan, R.O.C., in 1970 and M.S.E.E. and Ph.D. degrees from the University of California, Santa Barbara, in 1972 and 1975, respectively.

From 1970 to 1971, he was an engineering officer in the Chinese Navy Shipyard. From 1971 to 1975 , he was a Research Assistant at the University of California, Santa Barbara. He was the Professor and Chairman in the Electrical Engineering department of Tatung Institute of Technology and National Taiwan University from 1981 to 1983 and 1995 to 1998 , respectively. Presently, he is the Dean of Electrical Engineering and Computer Science College and the Professor of Electrical Engineering department at National Taiwan University. His research interests include digital signal processing, image processing, optical information processing, and laser holography.

Dr. Pei received the National Sun Yet-Sen Academic Achievement Award in Engineering in 1984, the Distinguished Research Award from the National Science Council from 1990 to 1998, the Outstanding Electrical Engineering Professor Award from the Chinese Institute of Electrical Engineering in 1998, the
Academic Achievement Award in Engineering from the Ministry of Education in 1998, the Pan Wen-Yuan Distinguished Research Award in 2002, and the National Chair Professor Award from Ministry of Education in 2002. He has been President of the Chinese Image Processing and Pattern Recognition Society in Taiwan from 1996 to 1998, and is a member of Eta Kappa Nu and the Optical Society of America (OSA). He became an IEEE Fellow in 2000 for contributions to the development of digital eigenfilter design, color image coding and signal compression, and to electrical engineering education in Taiwan.

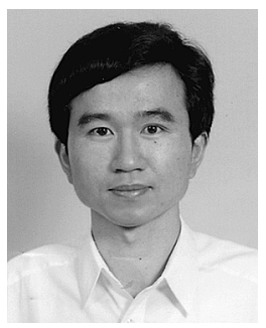

Wen-Liang Hsue was born in Taipei, Taiwan, R.O.C., in 1966. He received the B.S. and M.S. degrees, both in electrical engineering, from the National Taiwan University, Taipei, Taiwan, R.O.C., in 1988 and 1993, respectively. He is currently working towards the $\mathrm{Ph} . \mathrm{D}$. degree in communication engineering at the National Taiwan University.

From 1995 to 2000, he was with the Directorate General of Telecommunications, Taiwan, R.O.C. Since 2000, he has been a Lecturer in the Department of Electronic Engineering, Lan-Yang Institute of Technology, I-Lan, Taiwan. His current research interests include fractional Fourier transform, digital signal processing, and array signal processing.

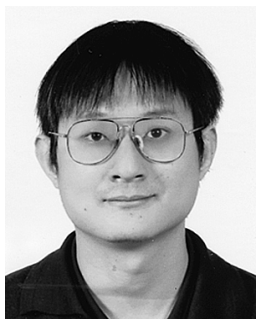

Jian-Jiun Ding was born in Taiwan, R.O.C., in 1973. He received the B.S., M.S., and Ph.D. degrees, all in electrical engineering, from the National Taiwan University (NTU), Taipei, Taiwan, R.O.C., in 1995 , 1997, and 2001, respectively.

Currently, he is a Postdoctoral Researcher with the Department of Electrical Engineering, NTU. His current research areas include fractional Fourier transforms, linear canonical transforms, orthogonal polynomials, fast algorithms, quaternion algebra, pattern recognition, and filter design. 